\title{
Topology of hypersurface singularities with 3-dimensional critical set
}

\author{
Javier Fernández de Bobadilla and Miguel Marco-Buzunáriz*
}

\begin{abstract}
In this paper, we prove that the Milnor fibre of a singularity over an isolated complete intersection singularity (i.c.i.s.) of dimension 3 has the homotopy type of a bouquet of spheres, provided that the function that defines the singularity has finite extended codimension with respect to the ideal that defines the i.c.i.s.
\end{abstract}

Mathematics Subject Classification (2010). 14J17, 32S25.

Keywords. Non-isolated singularities, Milnor fibration, morsification, bouquet theorems, deformations.

\section{Introduction}

In [13] Milnor introduced the Milnor fibration for any holomorphic germ

$$
f:\left(\mathbb{C}^{n}, O\right) \rightarrow \mathbb{C}
$$

and proved that the Milnor fibre is always a CW-complex of dimension at most $(n-1)$. In the case in which $f$ has an isolated singularity at the origin he also proved that the Milnor fibre is homotopy equivalent to a bouquet of $(n-1)$-spheres. The number of spheres is equal to the Milnor number $\mu$, which can be easily computed from the equation. If $f$ has non-isolated singularities at the origin the situation is much more complicated. Up to now, the only general result is the Kato-Matsumoto bound [10] which asserts that the Milnor fibre is $(s-2)$-connected, where $s$ is the codimension of the singular locus in $\mathbb{C}^{n}$. The homotopy type of the Milnor fibre of a general function germ can be very complicated. In fact, by a recent result of the first author [6], for any local analytic set in $\mathbb{C}^{m}$ there is a function whose Milnor fibre is homotopy equivalent to the complement of the set in a sufficiently small ball. The class of such spaces is very rich (contains for example the class of complements of hyperplane and line arrangements) and there is a whole theory dedicated to its study. Hence we may not

\footnotetext{
* Research partially supported by the ERC Starting Grant project TGASS and by Spanish Contract MTM200767908-C02-02. The authors thank the Facultad de Ciencias Matemáticas of the Universidad Complutense de Madrid for excellent working conditions.
} 
expect to find a simple description of the homotopy type of the Milnor fibre of a general function germ.

It is very interesting to find classes of non-isolated hypersurface singularities for which the homotopy type of the Milnor fibre admits an understandable description from the equation. This paper contributes to a program in this direction. Let $I \subset$ $\mathcal{O}_{\mathbb{C}^{n}, O}$ be an ideal defining a 3-dimensional i.c.i.s. $\Sigma_{0}$ and let $f$ be a function of finite extended codimension with respect to $I$ (see Section 2 for a definition). Our main results are the following:

(1) We prove that the Milnor fibre of $f$ is homotopic to a bouquet of spheres of different dimensions (see Theorem 11.2).

(2) We also compute the number of spheres appearing in terms of the equation (see Theorem 10.1).

Similar results for the cases in which $\Sigma_{0}$ is of dimension 1 and 2 were produced by the work of Siersma (see [18] and [19]), Zaharia [23] and Némethi [15]. If $\Sigma$ is a hypersurface the result was proved by Shubladze [17] and Némethi [14].

Actually we formulate the following:

Conjecture. The Milnor fibre of a function of finite extended codimension with respect to an i.c.i.s. has the homotopy type of a bouquet of spheres.

Functions of finite extended codimension with respect to an i.c.i.s. are a particular case of $I$-isolated singularities as defined and studied in [4]. There it was given a bouquet theorem decomposing homotopically the Milnor fibre in a bouquet of several $(n-1)$-spheres and an unknown space ([4], Theorem 9.3). The results of this paper identify the homotopy type of that space. It would be interesting to generalise this paper to other $I$-isolated singularities.

Other bouquet theorems in the context of singular ambient spaces were proved by Siersma [21] and Tibar [22].

Let us end with a description of some applications of this kind of results. The class of singularities studied in this paper shows very surprising phenomena from the equisingularity viewpoint. It has been used in [4] in order to disprove several old equisingularity questions. At the moment of writing the paper [4] some of the Betti number formula contained in this paper were known to the first author. It was this knowledge which lead him to guess the counterexamples contained in [4] (see Section 12 for more details). We hope that a systematic solution to our conjecture would lead to interesting examples showing other topological phenomena in nonisolated singularities yet unknown to us.

The structure of this paper is inspired in the classical Picard-Lefschetz theory of isolated singularities and Sierma's generalisation for non-isolated singularities. In this theory, a function is perturbed to split a singular point into several Morse-type singularities (this process is usually referred to as Morsification). Then it is shown that 
the homology of the Milnor fibre of the original function can be recovered from the Milnor fibres of each Morse-type singularity. Finally, these homologies are computed by a local study of the Morse-type singularities.

In Section 2 we use the results of [3] to prove that in our case we can do a process analogous to the Morsification, but instead of obtaining only Morse-type singularities, we will also obtain a non-isolated singularity over the Milnor fibre of the i.c.i.s. $\Sigma$. More precisely, we define a certain unfolding

$$
F: \mathbb{C}^{n} \times B \rightarrow \mathbb{C}
$$

of the function $f$ inside the ideal $I$ and a positive codimensional analytic subset $\Delta \subset B$ such that for any $b \in B \backslash \Delta$ the function $f_{b}:=F(., b)$ has as critical set the union of the Milnor fibre of the i.c.i.s. $\Sigma$ and finitely many Morse points. The non-isolated part of the critical set is stratified (in three pieces) according with the analytic type of the local singularity at each point. In this section we prove also some properties of the monodromy of this stratification around $\Delta$ which will be important for the rest of the paper.

In Section 3 we show that, as in the isolated case, the homology of the original Milnor fibre can be recovered from the pieces of the Milnor fibres contained in small neighbourhoods of the singularities obtained after the deformation. The procedure follows an idea of Siersma and is called homology splitting.

In Section 4 we study the topology of the local Milnor fibres of the singularity types that can appear in the generic deformation of $f$ within the ideal $I$.

Having done that, the hardest part of the paper is to study the part of the Milnor fibre contained in a tubular neighbourhood of the non-isolated singularity obtained after the deformation. Let us call this part of the Milnor fibre $\mathcal{M}$. This study is carried, roughly speaking decomposing $\mathcal{M}$ in pieces, studying their homology separately and gluing using Mayer-Vietoris sequences. It is hard to study the integral homology of the pieces directly, since non-orientable sphere-fibrations appear. This forces us to study first homology with coefficients in $\mathbb{Z}_{2}$ first, and lift to integral coefficients later.

The decomposition of $\mathcal{M}$ is based in the decomposition of the Milnor fibre of $\Sigma$ (the non-isolated part of the critical set of the deformation $f_{b}$ of $f$ ) by analytic type of the local singularity. In Section 5 these decompositions are described, and it is shown that each piece of $\mathcal{M}$ fibers naturally over the corresponding stratum of the Milnor fibre of $\Sigma$ with fibre a homotopy sphere.

In Section 6 we study the topology of a particular stratum of the stratification of the Milnor fibre of $\Sigma$. The study is somewhat involved, but it is a key step for the computation of the homology of the Milnor fibre of $f$.

In Sections 7 we study the homology of the part of the Milnor fibre corresponding to the stratum studied in the previous section. In Section 8 we use Mayer-Vietoris for a first time in order to study the homology of the union of two of the three pieces of $\mathcal{M}$. 
In Section 9 we complete the computation of the integral homology of $\mathcal{M}$. The first idea to run a Mayer-Vietoris sequence that glues the homology computed in the previous section with the homology of the remaining piece $\mathcal{M}$. However, as this seems complicated, we prove a key reduction, which allows to glue, instead of the remaining piece of $\mathcal{M}$, a collection of products of topological balls with spheres. This allows to determine the homology of $\mathcal{M}$ up to torsion and up to a direct summand isomorphic to $\mathbb{Z}$ in $H_{n-2}(\mathcal{M}, \mathbb{Z})$. We prove, using a differential form, that the direct summand appears or not depending on the value of a certain invariant of $f$. Finally we prove that the homology is torsion-free.

We end the paper computing in Sections 10 and 11 the homology and the homotopy type of the Milnor fibre of $f$. In particular we prove our conjecture for the case of 3-dimensional critical set.

The last section describes a distinguished family of functions belonging to the class studied in this paper which already had striking applications in topological equisingularity.

1.1. Terminology. If $X$ is a subspace of a topological space $Y$ we denote by $\dot{X}$ the interior points of $X$, and by $\partial X$ the boundary points of $X$ in $Y$. Given two topological spaces $X$ any $Y$ we denote that they have the same homotopy type by $X \simeq Y$. We will denote by $D_{\delta}$ the closed disc of radius $\delta$ in the complex plane and by $B_{\epsilon}$ the closed ball of radius $\epsilon$ in a complex affine space. The centers of the discs and balls will be clear from the context unless they are explicitly mentioned in the text or in the notation (by $B(x, \epsilon)$ ). Denote by $\mathbb{S}^{k}$ a sphere of dimension $k$. The zero set of an ideal $I$ will be denoted by $V(I)$, and $V\left(f_{1}, \ldots, f_{i}\right)$ will denote the zero set of the ideal generated by the polynomials $f_{1}, \ldots, f_{i}$. The origin of $\mathbb{C}^{i}$ will be denoted by $O$, being the dimension $i$ clear from the context. Given any finite set $K$ denote by $\operatorname{Aut}(K)$ its permutation group.

\section{Unfoldings}

In this section we will see how we can deform our function $f$, with critical locus $\Sigma_{0}$, into another one that has as singular locus the Milnor fibre $\Sigma$ of $\Sigma_{0}$, plus a certain number of isolated Morse points. We will also define a natural decomposition of $\Sigma$ that will be useful later.

Let $I:=\left(g_{1}, \ldots, g_{n-3}\right) \subset \mathcal{O}_{\mathbb{C}^{n}, O}$ be an ideal defining a 3-dimensional i.c.i.s. $\Sigma_{0}$ in $\left(\mathbb{C}^{n}, O\right)$. Denote by $\Theta_{I, e}$ the germs of vector fields tangent to the i.c.i.s., that is, the germs $X$ of vector fields such that $X(I)$ is included in $I$. A function-germ $f:\left(\mathbb{C}^{n}, O\right) \rightarrow \mathbb{C}$ is singular at $\Sigma$ if and only if it belongs to $I^{2}$. As in [16] we define the extended codimension of $f$ with respect to $I$ as

$$
c_{I, e}:=\operatorname{dim}_{\mathbb{C}}\left(I^{2} / \Theta_{I, e}(f)\right),
$$


where $\Theta_{I, e}(f)$ is the ideal whose elements are $X(f)$ for $X \in \Theta_{I, e}$. From the deformation viewpoint, functions with finite extended codimension play the same role in the space of functions singular in $\Sigma_{0}$ than isolated singularities in the space of all function-germs. A geometric characterisation of germs of finite extended codimension was given in [23] (see [4] for another proof and generalisations): these are germs in $I^{2}$ which outside the origin only have either isolated $A_{1}$ singularities or singularities of type $D(3, p)$, with $p \in\{0,1,2\}$.

The singularity $D(k, p)$ has the following normal form (see [16]):

$$
\sum_{1 \leq i \leq j \leq p} x_{i, j} y_{i} y_{j}+\sum_{p+1 \leq i \leq n-k} y_{i}^{2}=0
$$

where $\left\{x_{i, j}\right\}_{1 \leq i \leq j \leq p} \cup\left\{y_{i}\right\}_{1 \leq i \leq n-k}$ is an independent system of linear forms in $\mathbb{C}^{n}$.

Given a germ $f \in I^{2}$ we can express it as a matrix product

$$
f=\left(g_{1}, \ldots, g_{n-3}\right)\left(h_{i, j}\right)\left(g_{1}, \ldots, g_{n-3}\right)^{t}
$$

with $\left(h_{i, j}\right)$ a symmetric matrix of holomorphic germs of size $n-3$. An easy computation shows that the restriction $\left.\left(h_{i, j}\right)\right|_{\Sigma_{0}}$ only depends on $f$.

Let

$$
G_{1}, \ldots, G_{n-3}: \mathbb{C}^{n} \times B \rightarrow \mathbb{C}^{n-3}
$$

be the semiuniversal unfolding of the i.c.i.s. $\left(g_{1}, \ldots, g_{n-3}\right)$. Its base $B$ is a germ of complex manifold [11]. Given any $b \in B$ denote by

$$
\left(G_{1, b}, \ldots, G_{n-3, b}\right): \mathbb{C}^{n} \rightarrow \mathbb{C}^{n-3}
$$

the mapping corresponding to the parameter value $b$. In the $\operatorname{space} \operatorname{SM}(n-3)$ of symmetric matrices with complex entries we consider the stratification

$$
\operatorname{SM}(n-3)=\bigcup_{i=0}^{n-3} \operatorname{SM}(n-3, i),
$$

where $\operatorname{SM}(n-3, i)$ is the set of matrices of corank equal to $i$. Notice that $\overline{\operatorname{SM}(n-3, i)}$ consists of the set of matrices defined by the vanishing of the minors of size $n-2-i$. It is easy to check that $\operatorname{SM}(n-3, i)$ is of codimension $i(i+1) / 2$ in $\operatorname{SM}(n-3)$. We consider the unfolding

$$
F: \mathbb{C}^{n} \times B \times \operatorname{SM}(n-3) \rightarrow \mathbb{C}
$$

of the function $f$ defined by

$$
F\left(x_{1}, \ldots, x_{n}, b,\left(c_{i, j}\right)\right):=\left(G_{1, b}, \ldots, G_{n-3, b}\right)\left(h_{i, j}+c_{i, j}\right)\left(G_{1, b}, \ldots, G_{n-3, b}\right)^{t} .
$$


Notation 2.1. Denote by $S=B \times \operatorname{SM}(n-3)$ the base of the unfolding. Consider $\Sigma:=V\left(G_{1}, \ldots, G_{n-3}\right) \subset \mathbb{C}^{n} \times S$. Given any $s=\left(b,\left(c_{i, j}\right)\right) \in S$ we denote by $f_{s}: \mathbb{C}^{n} \rightarrow \mathbb{C}$ the function corresponding to the parameter value $s$, by $\Sigma_{s}$ the locus $V\left(G_{1, b}, \ldots, G_{n-3, b}\right)$ and by

$$
H(F): \Sigma \rightarrow \operatorname{SM}(n-3)
$$

the mapping defined by $H(F)\left(x, b,\left(c_{i, j}\right)\right):=\left(h_{i . j}(x)+c_{i, j}\right)$. Consider

$$
H\left(f_{s}\right):=H(F) \mid \Sigma_{s} .
$$

Define $\Sigma[i]:=H(F)^{-1}(\operatorname{SM}(n-3, i))$ and $\Sigma[i]_{s}:=H\left(f_{s}\right)^{-1}(\operatorname{SM}(n-3, i))$.

Figure 1 shows a schematic view of these sets.
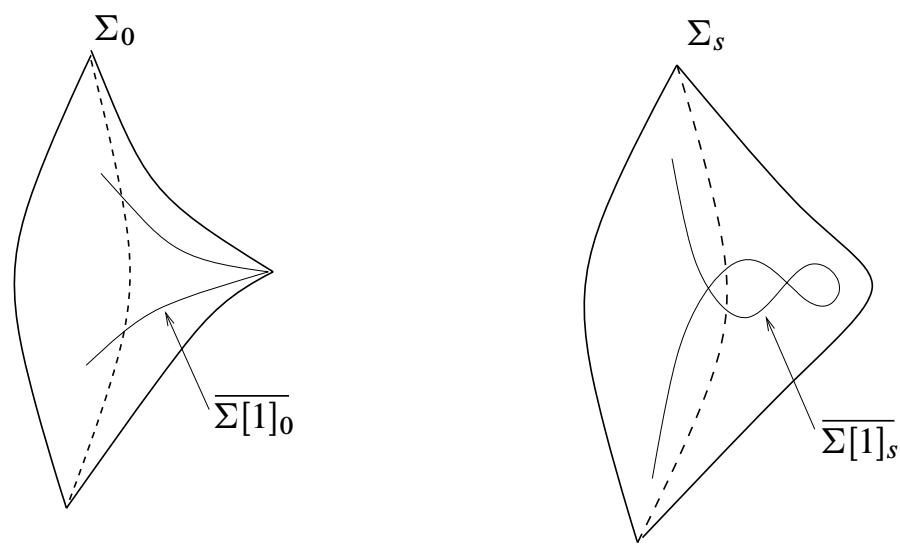

Figure 1. The deformation of the i.c.i.s. and the stratification.

The function $f_{0}$ coincides with $f$, where $0 \in S$ is the origin of the base of the unfolding.

Let $\epsilon$ and $\delta$ be radii for a Milnor fibration of $f$, that is radii such that

(1) the central fibre $f^{-1}(0)$ meets $\partial B_{\epsilon^{\prime}}$ transversely in the stratified sense for any $\epsilon^{\prime} \leq \epsilon$,

(2) for any $t \in D_{\delta} \backslash\{0\}$, the fibre $f^{-1}(t)$ meets $\partial B_{\epsilon}$ transversely,

(3) the only critical value of $\left.f\right|_{B_{\epsilon}}$ is 0 .

From [3] and [4] we obtain:

Theorem 2.2. There exists a proper closed analytic subset $\Delta$ of $S$, and a ball $B_{\eta}$ centred at $0 \in S$ such that for any $s \in B_{\eta} \backslash \Delta$ we have 
(1) for any $t \in D_{\delta}$ the intersection of $f_{s}^{-1}(t)$ with $\partial B_{\epsilon}$ is transversal (in the stratified sense if $t=0$ ).

(2) the critical set of the function $\left.f_{s}\right|_{B_{\epsilon}}$ is the union of $\Sigma_{S} \cap B_{\epsilon}$ with a finite number of Morse type singularities, whose critical values are pairwise different and different from 0 .

(3) the set $\Sigma_{s} \cap B_{\epsilon}$ is smooth (a Milnor fibre of the i.c.i.s. $\left(\Sigma_{0}, O\right)$ ) and the mapping

$$
\left.H\left(f_{S}\right)\right|_{\Sigma_{S} \cap B_{\epsilon}}: \Sigma_{s} \cap B_{\epsilon} \rightarrow \operatorname{SM}(n-3)
$$

is transversal to the stratification of $\operatorname{SM}(n-3)$ by corank. In particular $\Sigma[i]_{s}$ is a manifold of codimension $i(i+1) / 2$ in the 3-dimensional complex manifold $\Sigma_{s} \cap B_{\epsilon}$. Therefore the critical points of $f_{s}$ in $\Sigma_{s}$ are of type $D(3,0), D(3,1)$ or $D(3,2)$.

Denote by $\mathcal{C}$ and $\mathfrak{D}$ the critical set and the discriminant of the mapping

$$
\left(F, \mathrm{pr}_{2}\right): \mathbb{C}^{n} \times B_{\eta} \rightarrow \mathbb{C} \times B_{\eta},
$$

where $\mathrm{pr}_{2}$ denotes the projection of $\mathbb{C}^{n} \times B_{\eta}$ to the second factor. Then the restriction

$$
\left(F, \mathrm{pr}_{2}\right):\left(B_{\epsilon} \times B_{\eta}\right) \cap\left(F, \mathrm{pr}_{2}\right)^{-1}\left(\left(D_{\delta} \times B_{\eta}\right) \backslash \mathscr{D}\right) \rightarrow\left(D_{\delta} \times B_{\eta}\right) \backslash \mathscr{D}
$$

is a locally trivial fibration with fibre diffeomorphic to the Milnor fibre of $f$.

The set $\Delta$ is the subset of $S$ consisting of points $s$ where the critical set of $f_{s}$ is not as in (2).

Lemma 2.3. The set $\overline{\Sigma[1]_{0}}=V\left(\operatorname{det}(H(f)), g_{1}, \ldots, g_{n-3}\right)$ is a 2-dimensional i.c.i.s.

Proof. For any $s \in S$ the set of points of $\Sigma_{s} \cap B_{\epsilon}$ where $H\left(f_{S}\right)$ has corank at least 1 coincides with $V\left(\operatorname{det}\left(H\left(f_{s}\right)\right)\right.$. Denote by $c_{I_{x}, e}\left(\left(f_{s}\right)_{x}\right)$ the extended codimension of the germ $f_{s}$ at $x$ with respect to the ideal $I_{x}$ defining the germ $\left(\Sigma_{s}, x\right)$. In [3] it is shown that the set of points such that $H\left(f_{s}\right)$ is not transversal to the stratification of $\operatorname{SM}(n-3)$ by corank coincides precisely with the set of points at which $c_{I_{x}, e}\left(\left(f_{s}\right)_{x}\right)$ is non-zero.

Choose representatives $X_{1}, \ldots, X_{k}$ of the vector fields generating $\Theta_{I, e}$ defined on an open neighbourhood $W$ of the origin in $\mathbb{C}^{n}$. Then $c_{I, e}(f)$ is the dimension, as a complex vector space, of the stalk of the coherent sheaf of ideals in $\mathcal{O}_{\mathbb{C}}$ generated by $X_{1}(f), \ldots, X_{k}(f)$. Since this dimension is finite the origin is an isolated point in the support of the coherent sheaf of ideals. Consequently, the only point at which $H(f)$ is not transversal to the corank stratification is the origin if we take $\epsilon$ small enough.

Thus at any $x \in \Sigma_{0} \backslash\{0\}$ the germ $f_{x}$ is of type $D(3,0)$ if $\operatorname{det}(H(f)(x) \neq 0$ and of type $D(3,1)$ if $\operatorname{det}(H(f)(x)=0$. Inspecting the normal form of the $D(3,1)$ singularity we find that $V\left(\operatorname{det}(H(f)), g_{1}, \ldots, g_{n-3}\right)$ has an isolated singularity at the origin. 
Now we study the deformation $\overline{\Sigma[1]_{s}}:=V\left(\operatorname{det}\left(H\left(f_{s}\right)\right), G_{1, s}, \ldots, G_{n-3, s}\right) \cap B_{\epsilon}$ as we move in $S$. Choose $s \in S \backslash \Delta$. Since $H\left(f_{s}\right)$ is transversal to the stratification by corank there is a finite set of points $\Sigma[2]_{S}$ in $\Sigma[1]_{S}$ of type $D(3,2)$ and the rest of the points are of type $D(3,1)$. The normal form of the $D(3,2)$ singularity gives that $\overline{\Sigma[1]_{s}}$ has an $A_{1}$-type singularity at any point in $\Sigma[2]_{s}$. Let $a$ denote the cardinality of $\Sigma_{s}[2]$. In the next Lemma we show that a is independent on $s$.

Lemma 2.4. The restriction

$$
\operatorname{pr}_{2}:(\Sigma, \overline{\Sigma[1]}, \Sigma[2]) \cap \operatorname{pr}_{2}^{-1}(S \backslash \Delta) \rightarrow S \backslash \Delta
$$

is a topological locally trivial fibration of triples such that $\Sigma_{s}$ is the Milnor fibre of the i.c.i.s. $\Sigma_{0}$, the surface $\overline{\Sigma[1]_{s}}$ is a deformation of $\overline{\Sigma[1]_{0}}$ having precisely a singularities of $A_{1}$-type in $\Sigma[2]_{s}$. Moreover the restriction

$$
\operatorname{pr}_{2}: \Sigma[2] \cap \operatorname{pr}_{2}^{-1}(S \backslash \Delta) \rightarrow S \backslash \Delta
$$

is a covering and $\Sigma[2] \cap \operatorname{pr}_{2}^{-1}(S \backslash \Delta)$ is connected.

Proof. The topological triviality statements are easy to check from the normal forms of the $D(3, p)$ singularities.

The space $\Sigma$ is smooth since it is the product of the total space $\mathcal{V}$ of the versal deformation of the i.c.i.s. $V\left(g_{1}, \ldots, g_{n-3}\right)$ (which is smooth) with the space $\operatorname{SM}(n-3)$. For any matrix $M \in \operatorname{SM}(n-3)$ the fibre $H(F)^{-1}(M)$ is diffeomorphic to $\mathcal{V}$, being the diffeomorphism $\phi_{M}: \mathcal{V} \rightarrow H(F)^{-1}(M)$ defined by

$$
\phi_{M}(x):=\left(x, M-\left(h_{i, j}(x)\right) .\right.
$$

This shows that $H(F): \Sigma \rightarrow \operatorname{SM}(n-3)$ is a trivial fibration. The set $\Sigma[2]$ is connected since it is a Zariski dense open subset in the analytic manifold $H(F)^{-1}(\operatorname{SM}(n-3,2))$, which is diffeomorphic to the product $\mathcal{V} \times \operatorname{SM}(n-3,2)$, being $\operatorname{SM}(n-3,2)$ irreducible.

We summarise for further reference the main invariants introduced for the function $f$.

Definition 2.5. Define $\mu_{0}$ and $\mu_{1}$ to be the Milnor numbers at the origin of the i.c.i.s. $\Sigma_{0}$ and $\overline{\Sigma[1]_{0}}$. For $s \in S \backslash \Delta$ close to the origin we define $a$ to be the cardinality of $\Sigma[2]_{s}$ and $\# A_{1}$ to be the number of Morse points of $f_{s}$.

2.1. The corank $=2$ case. We will need an slightly larger unfolding of $f$ in the particular case in which corank $(H(f)(O))$ is precisely equal to 2 . In that case, after possibly changing the generators of the i.c.i.s we can assume that $f$ is of the form

$$
f=\left(g_{1}, g_{2}\right)\left(h_{i, j}\right)\left(g_{1}, g_{2}\right)^{t}+\sum_{i=3}^{n-3} g_{i}^{2} \text {. }
$$


When $c_{I, e}(f)$ is finite, the mapping

$$
H(f): \Sigma \rightarrow \operatorname{SM}(2)
$$

is transverse to the corank stratification outside the origin. Hence the origin is an isolated point of the locus where corank $(H(f))$ is at least 2 . Since in this case the corank 2 locus is defined by the vanishing of the $n$ functions $h_{1,1}, h_{1,2}, h_{2,2}, g_{1}, \ldots, g_{n-3}$, we have that $\Sigma[2]_{0}$ is a 0 -dimensional i.c.i.s. concentrated at the origin. Let a be its length as 0-dimensional scheme. Let

$$
\Sigma[2]=V\left(H_{1,1}, H_{1,2}, H_{2,2}, G_{1}, \ldots, G_{n-3}\right) \subset \mathbb{C}^{n} \times S \rightarrow S
$$

be the versal deformation of the i.c.i.s. $V\left(h_{1,1}, h_{1,2}, h_{2,2}, g_{1}, \ldots, g_{n-3}\right)$. Any fibre $\Sigma[2]_{s}$ is a 0 -dimensional scheme of length $a$. The discriminant $\Lambda \subset S$ is the set of parameters where $\Sigma[2]_{s}$ is non-reduced. By versality the discriminant is irreducible and reduced (Corollary 4.11 and Proposition 6.11 of [11]), and its smooth locus $\dot{\Lambda}$ is the set of parameters such that $\Sigma[2]_{s}$ has exactly a fat point of length 2 and is otherwise reduced (Lemma 4.9 of [11]).

Definition 2.6. Fix a base point $s_{0} \in S \backslash \Lambda$. Any path $\gamma:[0,1] \rightarrow S$ such that $\gamma(0)=s_{0}, \gamma([0,1))$ is included in $S \backslash \Lambda$ and $\gamma(1)$ is a smooth point of $\Lambda$ induces a deformation $\left\{\Sigma[2]_{t}\right\}_{t \in[0,1]}$ along $\gamma$ such that precisely two points $\left\{p_{0}, p_{1}\right\}$ in $\Sigma[2]_{0}=$ $\Sigma[2]_{s_{0}}$ collapse to the same point in $\Sigma[2]_{1}$. The vanishing cycle in $\Sigma[2]_{s_{0}}$ associated to $\gamma$ is, by definition, the pair $\left\{p_{0}, p_{1}\right\}$.

Lemma 2.7. All the points of $\Sigma[2]_{s_{0}}$ are at the same equivalence class by the equivalence relation generated by the vanishing cycles.

Proof. The base $S$ of the versal unfolding

$$
\psi: \Sigma[2] \rightarrow S
$$

of the 0 -dimensional i.c.i.s. $\Sigma[2]_{0}$ can be identified with a neighbourhood $U$ of the origin in $\mathbb{C}^{N}$. We choose a straight line $l$ through $s_{0}$ such that $l$ meets $\Lambda$ transversely at smooth points. The neighbourhood and the line can be chosen so that $\psi^{-1}(l \cap U)$ is the Milnor fibre of a 1-dimensional i.c.i.s. Therefore $\psi^{-1}(l \cap U)$ is connected ([11], Chapter 5). Choose a system of paths $\left\{\gamma_{i}\right\}_{i=1}^{M}$ joining $s_{0}$ with each one of the points of $l \cap \Lambda$, without self intersections and not intersecting pairwise except at $s_{0}$. Since the space $\psi^{-1}(l \cap U)$ is homotopy equivalent to the result of attaching a 1-cell at each one of the vanishing cycles associated to the paths $\left\{\gamma_{i}\right\}_{i=1}^{M}$, the connectivity of $\psi^{-1}(l \cap U)$ proves the lemma.

Consider $\operatorname{Sym}^{2}\left(\Sigma[2]_{s_{0}}\right)$ the second symmetric product of $\Sigma[2]_{s_{0}}$; and denote by $\mathfrak{D}$ its diagonal. Then $\operatorname{Sym}^{2}\left(\Sigma[2]_{s_{0}}\right) \backslash \mathfrak{D}$ is the set of subsets of cardinality precisely 2 . The monodromy action

$$
\rho: \pi_{1}\left(S \backslash \Lambda, s_{0}\right) \rightarrow \operatorname{Aut}\left(\Sigma[2]_{s_{0}}\right)
$$


induces a monodromy action

$$
\rho_{2}: \pi_{1}\left(S \backslash \Lambda, s_{0}\right) \rightarrow \operatorname{Aut}\left(\operatorname{Sym}^{2}\left(\Sigma[2]_{s_{0}}\right) \backslash \mathfrak{D} .\right.
$$

The set of vanishing cycles is a subset of $\operatorname{Sym}^{2}\left(\Sigma[2]_{s_{0}}\right) \backslash \mathfrak{D}$.

Lemma 2.8. The monodromy action $\rho_{2}$ preserves the set of vanishing cycles and acts transitively on it. In other words, the set of vanishing cycles is an orbit by the monodromy action.

Proof. A vanishing cycle induced by a path $\gamma$ is transformed by an element $[\alpha] \epsilon$ $\pi_{1}\left(S \backslash \Lambda, s_{0}\right)$ to the vanishing cycles induced by the concatenation path $\alpha \cdot \gamma$. The transitivity is a classical consequence of the irreducibility of the discriminant ([1], Chapter 3).

We enlarge the unfolding of $f$ defined in (1) by considering the following one instead of it:

$$
F: \mathbb{C}^{n} \times S \rightarrow \mathbb{C}
$$

given by

$$
F:=\left(G_{1}, G_{2}\right)\left(H_{i, j}\right)\left(G_{1}, G_{2}\right)^{t}+\sum_{i=3}^{n-3} G_{i}^{2} .
$$

The statements of Theorem 2.2 and Lemma 2.3 clearly remain true for this unfolding.

\section{Homology splitting}

In this section we will follow a general method of Siersma [18], [19], [20] to see that the Milnor fibre of the previous unfolding has the same homology than the Milnor fibre of the original function $f$. We will also see that the computation of this homology can be reduced to the computation of the homology of the part of the Milnor fibre that lies in a small neighbourhood of $\Sigma$.

We have chosen radii $\epsilon$ and $\delta$ for a Milnor fibration of $f$. In that situation the total space $X_{0}:=B_{\epsilon} \cap f^{-1}\left(D_{\delta}\right)$ of the representative

$$
f: X_{0} \rightarrow \mathbb{C}
$$

is contractible.

Consider the versal unfolding $F: \mathbb{C}^{n} \times S \rightarrow \mathbb{C}$ defined in the previous section. Choose a direction in $S$ such that the line $l$ through the origin $O$ of $S$ in this direction 
has $O$ as an isolated point of $\Delta$. Let $D_{\xi}$ be a disc in $l$ around $O$ only meeting $\Delta$ in $O$. Consider the associated 1-parameter unfolding

$$
F: \mathbb{C}^{n} \times D_{\xi} \rightarrow \mathbb{C} .
$$

Denote by $f_{s}$ the function $f_{s}(x):=F(x, s)$. By Ehresmann fibration theorem $X_{s}:=B_{\epsilon} \cap f_{s}^{-1}\left(D_{\eta}\right)$ is diffeomorphic to $X_{0}$ and hence it is contractible. If $s \neq 0$ the function

$$
f_{s}: X_{s} \rightarrow D_{\delta}
$$

is a locally trivial fibration over $D_{\delta} \backslash\left\{0, v_{1}, \ldots, v_{r}\right\}$, where $\left\{0, v_{1}, \ldots, v_{r}\right\}$ are the critical values of $f_{s}$. Each $v_{i} \neq 0$ is the image of precisely one singular point of type $A_{1}$ of $f_{s}$. The fibre of $f_{s}$ over any point $w$ not in $\left\{0, v_{1}, \ldots, v_{r}\right\}$ is diffeomorphic to the Milnor fibre of $f$. Therefore we are interested in the reduced homology $\widetilde{H}_{k}\left(f_{s}^{-1}(w) ; \mathbb{Z}\right)$, which is isomorphic to $H_{k+1}\left(X_{s}, f_{s}^{-1}(w) ; \mathbb{Z}\right)$ by the contractibility of $X_{s}$.

Consider $D_{0}, D_{1}, \ldots, D_{r}$ a system of disjoint small disks inside $D_{\delta}$ centered in $0, v_{1}, \ldots v_{k}$ respectively. Choose points $t_{i} \in \partial D_{i}$, and disjoint paths $\alpha_{i}$ joining $t_{0}$ with $t_{i}$. We can take $w=t_{0}$. Define

$$
G:=\bigcup_{i=1}^{r} \alpha_{i} \cup \bigcup_{i=0}^{r} D_{i} .
$$

It is clear that $G$ is a deformation retract of $D_{\delta}$, and since $f_{s}$ is a locally trivial fibration outside $G$, we have that

$$
H_{k}\left(X_{s}, f_{s}^{-1}(w)\right)=H_{k}\left(f_{s}^{-1}(G), f_{s}^{-1}(w)\right) .
$$

By excision,

$$
H_{k}\left(f_{t}^{-1}(G), f_{s}^{-1}(w)\right)=\bigoplus_{i=0}^{r} H_{k}\left(f_{s}^{-1}\left(D_{i}\right), f_{s}^{-1}\left(t_{i}\right)\right) .
$$

It is classical from Picard-Lefschetz theory that for any $i>0$ we have

$$
H_{n}\left(f_{s}^{-1}\left(D_{i}\right), f_{s}^{-1}\left(t_{i}\right)\right) \cong \mathbb{Z}
$$

and

$$
H_{k}\left(f_{s}^{-1}\left(D_{i}\right), f_{s}^{-1}\left(t_{i}\right)\right)=0
$$

if $k \neq n$.

Now let $T$ be a tubular neighbourhood of $\Sigma_{s}$. We can take for example $T:=$ $\left(G_{1, s}, \ldots, G_{n-3, s}\right)^{-1}(B)$ for $B$ a small ball around the origin in $\mathbb{C}^{n-3}$. Taking $T$ small enough and $D_{0}$ small in comparison with $T$ we can assume that $f_{s}^{-1}(t)$ meets the boundary $\partial T$ transversely for any $t \in D_{0}$. We define

$$
\mathcal{M}:=f_{s}^{-1}\left(t_{0}\right) \cap T \text {. }
$$


By this tranversality, and because of the fact that $f_{s}$ has no critical points in $f_{s}^{-1}\left(D_{0}\right) \backslash T$, the part of the space $f_{s}^{-1}\left(D_{0}\right)$ that lives outside $T$ can be retracted to $f_{s}^{-1}\left(t_{0}\right)$. This means that the pair $\left(f_{s}^{-1}\left(D_{0}\right), f_{s}^{-1}\left(t_{0}\right)\right)$ is homotopy equivalent to the pair $\left(f_{s}^{-1}\left(t_{0}\right) \cup T, f_{s}^{-1}\left(t_{0}\right)\right)$. By excision we have

$$
H_{k}\left(f_{s}^{-1}\left(D_{0}\right), f_{s}^{-1}\left(t_{0}\right)\right) \cong H_{k}\left(T, f_{s}^{-1}\left(t_{0}\right) \cap T\right)=H_{k}(T, \mathcal{M}) .
$$

We summarise what we have obtained:

Proposition 3.1. Denote the Milnor fibre of $f$ by $\mathbf{F}_{f}$. Let $r$ be the number of $A_{1}$ points that $f_{s}$ has in $B_{\epsilon}$ for $s \in S \backslash \Delta$ close to the origin of $S$. Then

$$
\begin{aligned}
H_{n-1}\left(\mathbf{F}_{f} ; \mathbb{Z}\right) & \cong H_{n}(T, \mathcal{M} ; \mathbb{Z}) \oplus \mathbb{Z}^{r}, \\
H_{k}\left(\mathbf{F}_{f} ; \mathbb{Z}\right) & \cong H_{k+1}(T, \mathcal{M} ; \mathbb{Z})
\end{aligned}
$$

for $1 \leq k \leq n-2$. By connectivity

$$
H_{0}\left(\mathbf{F}_{f} ; \mathbb{Z}\right) \cong \mathbb{Z}
$$

By construction, $T$ is homotopic to $\Sigma_{s}$, which is the Milnor fibre of $\Sigma_{0}$. We will spend a large part of this paper computing the homology of $\mathcal{M}$.

\section{The Milnor fibre of the $D(k, p)$ singularity}

We dedicate this section to study the local topology of a certain type of singularities that will appear later. We collect the following proposition, which follows from [5] and [7]. We reprove it here since the proof is crucial for the understanding of this paper.

Proposition 4.1. The Milnor fibre of the $D(k, p)$ singularity in $\mathbb{C}^{n}$ is homotopyequivalent to the sphere $\mathbb{S}^{n+p-k-1}$.

Proof. Since the singularity $D(k, p)$ is quasi-homogeneous its Milnor fibre is diffeomorphic to the global hypersurface $X \subset \mathbb{C}^{n}$ defined by

$$
\sum_{1 \leq i \leq j \leq p} x_{i, j} y_{i} y_{j}+\sum_{p+1 \leq i \leq n-k} y_{i}^{2}=1,
$$

where $\left\{x_{i, j}\right\}_{1 \leq i \leq j \leq p} \cup\left\{y_{i}\right\}_{1 \leq i \leq n-k}$ is an independent system of linear forms in $\mathbb{C}^{n}$. Hence $X$ is homotopic to the $(n-p-k)$-suspension of the hypersurface $Y \subset \mathbb{C} \frac{p^{2}+3 p}{2}$ defined by

$$
\sum_{1 \leq i \leq j \leq p} x_{i, j} y_{i} y_{j}=1
$$


The projection

$$
\sigma: Y \rightarrow \mathbb{C}^{k} \backslash\{O\}
$$

defined by $\sigma\left(x_{i, j}, y_{l}\right):=y_{l}$ is a locally trivial fibration with fibre an affine hyperplane in $\mathbb{C}^{\frac{p^{2}+p}{2}}$, and hence contractible. We conclude that $Y$ is homotopy equivalent to the unit sphere $\mathbb{S}^{2 k-1}$ in $\mathbb{C}^{k}$. Consequently $X$ is homotopic to the sphere $\mathbb{S}^{n+k-p-1}$.

Lemma 4.2. Given the $D(1,1)$ singularity

$$
f:=x_{1} y_{2}^{2}+y_{3}^{2}+\cdots+y_{n}^{2}:\left(\mathbb{C}^{n}, O\right) \rightarrow \mathbb{C}
$$

in $\mathbb{C}^{n}$ its restriction

$$
\left.f\right|_{H_{1}}: H_{1} \rightarrow \mathbb{C}
$$

to the hyperplane $H_{1}$ defined by $x_{1}=1$ is a Morse singularity at the origin. The pair of Milnor fibres $\left(f^{-1}(t),\left(\left.f\right|_{H_{1}}\right)^{-1}(t)\right)$ is homotopy equivalent to the pair $\left(\mathbb{S}^{n-1}, \mathbb{S}^{n-2}\right)$ with $\mathbb{S}^{n-2}$ embedded in $\mathbb{S}^{n-1}$ as the equator.

Proof. By suspension it is enough to consider $n=2$, and in this case it is obvious, since the Milnor fibre of $x_{1} y_{2}^{2}$ projects to the double cover of $\mathbb{C} \backslash\{O\}$ by the projection $\operatorname{pr}(x, y)=x$ and the Milnor fibre of $\left.f\right|_{H_{1}}$ is a fibre of this projection.

It is necessary for the rest of the paper to collect further geometric facts about the Milnor fibration of the $D(3,2)$ singularity and its interaction with the Milnor fibration of the transversal singularities through points of its critical set which are close to the origin. Such transversal singularities are of type $D(1,1)$ at the points different from the origin where $\operatorname{det}(H(f)$ vanishes and of Morse type at the generic points. We do this in the what follows.

Consider the $D(3,2)$ singularity

$$
f:=x_{1} y_{1}^{2}+2 x_{2} y_{1} y_{2}+x_{3} y_{2}^{2}+y_{3}^{2}+\cdots+y_{n-3}^{2}:\left(\mathbb{C}^{n}, O\right) \rightarrow \mathbb{C}
$$

in $\mathbb{C}^{n}$

Recall from Notation 2.1 the definition of $\Sigma[i]$. As here we are considering no unfolding we have the equality $\Sigma=\Sigma_{0}$. The restriction of $f$ to any $(n-3)$ dimensional transversal to $\Sigma[0]$ is a Morse type singularity in $\mathbb{C}^{n-3}$. The restriction of $f$ to any $(n-2)$-dimensional transversal to $\Sigma[1]$ is a $D(1,1)$ singularity in $\mathbb{C}^{n-2}$.

The stratum $\Sigma[1]$ is equal to $V(\operatorname{det}(H(f)) \backslash\{0\}$ and hence is homotopic to its link $L_{\epsilon}:=\Sigma[1] \cap \mathbb{S}_{\epsilon}$ at the origin. Since the singularity is homogeneous we can take $\epsilon=1$ and denote $L_{\epsilon}$ by $L$. This link is diffeomorphic to $\mathbb{R P}^{3}$, since the surface $\Sigma[1]$ is defined by $\operatorname{det}(H(f))\left(x_{1}, x_{2}, x_{3}\right)=x_{1} x_{3}-x_{2}^{2}=0$ in $\Sigma \cong \mathbb{C}^{3}$. Hence its fundamental group is isomorphic to $\mathbb{Z}_{2}$. 
Let

$$
\kappa: N\left(\mathbb{C}^{n}, \Sigma\right) \rightarrow \Sigma
$$

and

$$
\kappa_{1}: N\left(\mathbb{C}^{n}, \Sigma[1]\right) \rightarrow \Sigma[1]
$$

be the holomorphic normal bundles of $\Sigma$ and $\Sigma[1]$ in $\mathbb{C}^{n}$ respectively. We have the inclusion of restrictions

$$
\iota:\left.N\left(\mathbb{C}^{n}, \Sigma\right)\right|_{\Sigma[1]} \hookrightarrow N\left(\mathbb{C}^{n}, \Sigma[1]\right),
$$

compatible with the bundle maps.

Observe that, since in this case $\Sigma$ is a 3-dimensional coordinate subspace, the first bundle is trivial with fibre $\mathbb{C}^{n-3}$. Notice that, as $L$ is compact, there is a positive $\rho$ such that the $\rho$-neighbourhood of the zero section of the restriction

$$
\left.\kappa_{1}\right|_{L}: N\left(\mathbb{C}^{n}, \Sigma[1]\right)_{L} \rightarrow L
$$

embeds in $\mathbb{C}^{n}$ holomorphically on each fibre. We denote by $\left.N^{\rho}\left(\mathbb{C}^{n}, \Sigma[1]\right)\right|_{L}$ this $\rho$-neighbourhood, and by

$$
\kappa_{1}^{\rho}:\left.N^{\rho}\left(\mathbb{C}^{n}, \Sigma[1]\right)\right|_{L} \rightarrow L
$$

its embedding; its fibre is a complex $(n-2)$-dimensional ball.

For any $y \in L$ the restriction of $f$ to the fibre of the embedded normal bundle

$$
\left.f\right|_{N^{\rho}\left(\mathbb{C}^{n}, \Sigma[1]\right)_{y}}:\left(N^{\rho}\left(\mathbb{C}^{n}, \Sigma[1]\right)_{y}, y\right) \rightarrow \mathbb{C}
$$

is a $D(1,1)$ singularity with critical set the line $\operatorname{Crit}(y):=N^{\rho}\left(\mathbb{C}^{n}, \Sigma[1]\right) y \cap \Sigma$.

Since the restriction of the function $\operatorname{det}(H(f))$ to $\operatorname{Crit}(y)$ is non-singular at the point $y$ for any $y \in L$, for $u$ small enough the intersection $\operatorname{Crit}(y) \cap \operatorname{det}(H(f))^{-1}(u)$ is a unique point for any $y \in L$, and hence

$$
\Xi_{u}:=\left.\operatorname{det}(H(f))^{-1}(u) \cap \Sigma \cap N^{\rho}\left(\mathbb{C}^{n}, \Sigma[1]\right)\right|_{L}
$$

defines a cross-section of the embedded normal bundle, and the restriction

$$
\left.\kappa_{1}^{\rho}\right|_{\Xi_{u}}: \Xi_{u} \rightarrow L
$$

is a diffeomorphism.

Let

$$
\kappa^{\rho}:\left.N^{\rho}\left(\mathbb{C}^{n}, \Sigma\right)\right|_{\Xi_{u}} \rightarrow \Xi_{u}
$$

be a holomorphic embedding of a $\rho$-neighbourhood of the zero section of the restriction to $\Xi_{u}$ of the normal bundle of $\Sigma$ in $\mathbb{C}^{n}$. It is a trivial bundle with fibre a 
complex $(n-3)$-dimensional ball. For any $x \in \Xi_{u}$ the restriction of $f$ to the fibre $\left(N^{\rho}\left(\mathbb{C}^{n}, \Sigma\right) \mid \Xi_{u}\right)_{x}$ is a Morse type singularity.

If $\rho$ and $u$ are chosen small enough we may assume that, for any $x \in \Xi_{u}$, we have an inclusion of fibres

$$
\left(\left.N^{\rho}\left(\mathbb{C}^{n}, \Sigma\right)\right|_{\Xi_{u}}\right)_{x} \subset\left(N\left(\mathbb{C}^{n}, \Sigma[1]\right)_{L}\right)_{\left.\kappa_{1}^{\rho}\right|_{\Xi_{u}}(x)}
$$

Now we study the restrictions of the bundle maps $\kappa^{\rho}$ and $\kappa_{1}^{\rho}$ to a fibre of $f^{-1}(\delta)$ for small $\delta$. Define

$$
\begin{aligned}
& \alpha:=\left.\kappa_{1}^{\rho}\right|_{\left.f^{-1}(\delta) \cap N^{\rho}\left(\mathbb{C}^{n}, \Sigma[1]\right)\right|_{L}}:\left.f^{-1}(\delta) \cap N^{\rho}\left(\mathbb{C}^{n}, \Sigma[1]\right)\right|_{L} \rightarrow L, \\
& \beta:=\left.\left.\kappa_{1}^{\rho}\right|_{\Xi_{u}} \circ \kappa^{\rho}\right|_{f^{-1}(\delta) \cap N^{\rho}\left(\mathbb{C}^{n}, \Sigma\right) \mid \Xi_{u}}:\left.f^{-1}(\delta) \cap N^{\rho}\left(\mathbb{C}^{n}, \Sigma\right)\right|_{\Xi_{u}} \rightarrow L .
\end{aligned}
$$

We have

Lemma 4.3. For $\delta$ small enough the mapping

$$
(\alpha, \beta):\left(\left.f^{-1}(\delta) \cap N^{\rho}\left(\mathbb{C}^{n}, \Sigma[1]\right)\right|_{L},\left.f^{-1}(\delta) \cap N^{\rho}\left(\mathbb{C}^{n}, \Sigma\right)\right|_{\Xi_{u}} \rightarrow L\right.
$$

is a locally trivial fibration of pairs with fibre homotopic to $\left(\mathbb{S}^{n-3}, \mathbb{S}^{n-4}\right)$, with $\mathbb{S}^{n-4}$ embedded in $\mathbb{S}^{n-3}$ as an equator and whose monodromy is isotopic to the identity in $\mathbb{S}^{n-4}$ and it is the reflection over the equator in $\mathbb{S}^{n-3}$.

Proof. The statement of the homotopy type of the fibre is just Lemma 4.2.

The circle $\gamma(\theta):=\left(0,0, e^{2 \pi i \theta}\right)$ parametrises a generator of the fundamental group of $L$. The normal bundle $\left.N^{\rho}\left(\mathbb{C}^{n}, \Sigma[1]\right)\right|_{L}$ can be chosen so that for any $\theta$ the line $\operatorname{Crit}(\gamma(\theta))$ is equal to $V\left(x_{2}, x_{3}-e^{2 \pi i \theta}, y_{1}, \ldots, y_{n-3}\right)$ and the cross-section $\Xi_{u}$ is defined by $\Xi_{u}(\gamma(\theta))=\left(u e^{-2 \pi i \theta}, 0, e^{2 \pi i \theta}, 0, \ldots, 0\right)$.

For any $\theta \in[0,2 \pi]$ the pair of fibres $\left(\alpha^{-1}(\gamma(\theta)), \beta^{-1}(\gamma(\theta))\right)$ is homotopic to the pair of varieties $\left(X_{\theta}, Y_{\theta}\right)$ defined by

$$
\begin{gathered}
X_{\theta}:=V\left(x_{2}, x_{1} y_{1}^{2}+e^{2 \pi i \theta} y_{2}^{2}+y_{3}^{2}+\cdots+y_{n-3}^{2}\right), \\
Y_{\theta}:=V\left(x_{2}, u e^{-2 \pi i \theta} y_{1}^{2}+e^{2 \pi i \theta} y_{2}^{2}+y_{3}^{2}+\cdots+y_{n-3}^{2}, x_{1}-u e^{-2 \pi i \theta}\right) .
\end{gathered}
$$

The family of diffeomorphism

$$
\varphi_{\theta}: \mathbb{C}^{n} \rightarrow \mathbb{C}^{n}
$$

defined by

$\varphi_{\theta}\left(x_{1}, x_{2}, x_{3}, y_{1}, \ldots, y_{n-3}\right):=\left(e^{-2 \pi i \theta} x_{1}, x_{2}, x_{3}, e^{\pi i \theta} y_{1}, e^{-\pi i \theta} y_{2}, y_{3}, \ldots, y_{n-3}\right)$

induces a diffeomorphism from $\left(X_{0}, Y_{0}\right)$ to $\left(X_{\theta}, Y_{\theta}\right)$ for any $\theta \in[0,2 \pi]$. Therefore a geometric monodromy is given by

$$
\varphi_{1}:\left(X_{0}, Y_{0}\right) \rightarrow\left(X_{1}, Y_{1}\right)=\left(X_{0}, Y_{0}\right) .
$$

The pair $\left(X_{0}, Y_{0}\right)$ is homotopic to $\left(\mathbb{S}^{n-3}, \mathbb{S}^{n-4}\right)$ and it is easy to check that $\varphi_{1}$ preserves the orientation in $\mathbb{S}^{n-4}$ and reverses it in $\mathbb{S}^{n-3}$. 


\section{A decomposition of $\mathcal{M}$}

In this section we will define a decomposition of $\mathcal{M}$ in several pieces. This decomposition is defined after the stratification of $\Sigma_{s}$ defined in Section 2. The topology of each piece, and the gluing of them will be studied in the following sections.

In Proposition 3.1 it has become clear that in order to understand the homology of the Milnor fibre of $f$ we need to compute the homology of the intersection $\mathcal{M}=$ $f_{s}^{-1}\left(t_{0}\right) \cap T$, with $s \in S \backslash \Delta$ small enough and $t_{0} \neq 0$ small enough. The tubular neighbourhood $T$ is the total space of a trivial fibration

$$
\pi: T \rightarrow \Sigma_{s}
$$

with fibre an $(n-3)$-complex dimensional ball. If $B$ is a subspace of $\Sigma_{s}$ we denote $\pi^{-1}(B)$ by $T_{B}$.

By Theorem 2.2, for a generic parameter $s$ close to the origin of the base $S$ of the unfolding of $f$, the maximal corank of $H\left(f_{s}\right)(x)$ is two for any $x \in \Sigma_{s}$. Recall that the set of points where the corank is at least 1 is the surface $\overline{\Sigma[1]_{s}}$ defined by the vanishing of $\operatorname{det}\left(H\left(f_{s}\right)\right)$. The singular points $\Sigma[2]_{s}=\left\{p_{1}, \ldots, p_{a}\right\}$ of $\overline{\Sigma[1]_{s}}$ are of Morse type and coincide precisely with the points where the corank of $H\left(f_{s}\right)$ equals 2 .

For each point $p_{i}$ let $B_{i}(\zeta)$ be a ball of radius $\zeta$ around $p_{i}$ in $\mathbb{C}^{n}$ such that $\left.f_{s}\right|_{B_{i}}(\zeta)$ is biholomorphic to the restriction of the singularity $D(3,2)$ to the unit ball of $\mathbb{C}^{n}$. Taking $\zeta$ small enough we can assume that the balls are mutually disjoint and that the intersections $A_{i}(\zeta):=B_{i}(\zeta) \cap \Sigma_{s}$ are balls in $\Sigma_{s}$ centered in each of the points $p_{i}$. Taking $T, \zeta, s$, and $t_{0}$ small enough the space

$$
\mathcal{A}_{i}:=f_{s}^{-1}\left(t_{0}\right) \cap \pi^{-1}\left(A_{i}(\zeta)\right)=\mathcal{M} \cap \pi^{-1}\left(A_{i}(\zeta)\right)
$$

is diffeomorphic to the Milnor fibre of $f_{s}$ at $p_{i}$ for any $i$, and hence homotopy equivalent to $\mathbb{S}^{n-2}$ (see Lemma 4.1).

Now we choose the following parameters:

- We take $\zeta$ small enough so that $\partial A_{i}\left(\zeta^{\prime}\right)$ is transverse to $\overline{\Sigma[1]_{s}}$ for any $0<\zeta^{\prime}<\zeta$.

- We choose $\zeta_{0}<\zeta$ sufficiently close to $\zeta$ so that the inclusion

$$
\mathcal{M} \cap \pi^{-1}\left(A_{i}\left(\zeta^{\prime}\right)\right) \subset \mathcal{A}_{i}
$$

is a homotopy equivalence for any $\zeta_{0} \leq \zeta^{\prime} \leq \zeta$.

- Choose $\xi>0$ small enough so that $\operatorname{det}\left(H\left(f_{s}\right)\right)^{-1}(u)$ meets $\partial A_{i}\left(\zeta^{\prime}\right)$ transversely for any $u \in D_{\xi}$ and $\zeta_{0} \leq \zeta^{\prime} \leq \zeta$.

Consider $\dot{A}_{i}\left(\zeta_{0}\right)$ the interior of $A_{i}\left(\zeta_{0}\right)$ and define

$$
B:=\operatorname{det}\left(H\left(f_{s}\right)\right)^{-1}\left(D_{\xi}\right) \backslash\left(\bigcup_{i=1}^{a} \dot{A}_{i}\left(\zeta_{0}\right)\right),
$$


Vol. 88 (2013) Topology of hypersurface singularities with 3-dimensional critical set

$$
B_{u}:=\operatorname{det}\left(H\left(f_{s}\right)\right)^{-1}(u) \backslash\left(\bigcup_{i=1}^{a} \dot{A}_{i}\left(\zeta_{0}\right)\right) .
$$

A schematic picture of this decomposition can be seen in Figure 2.

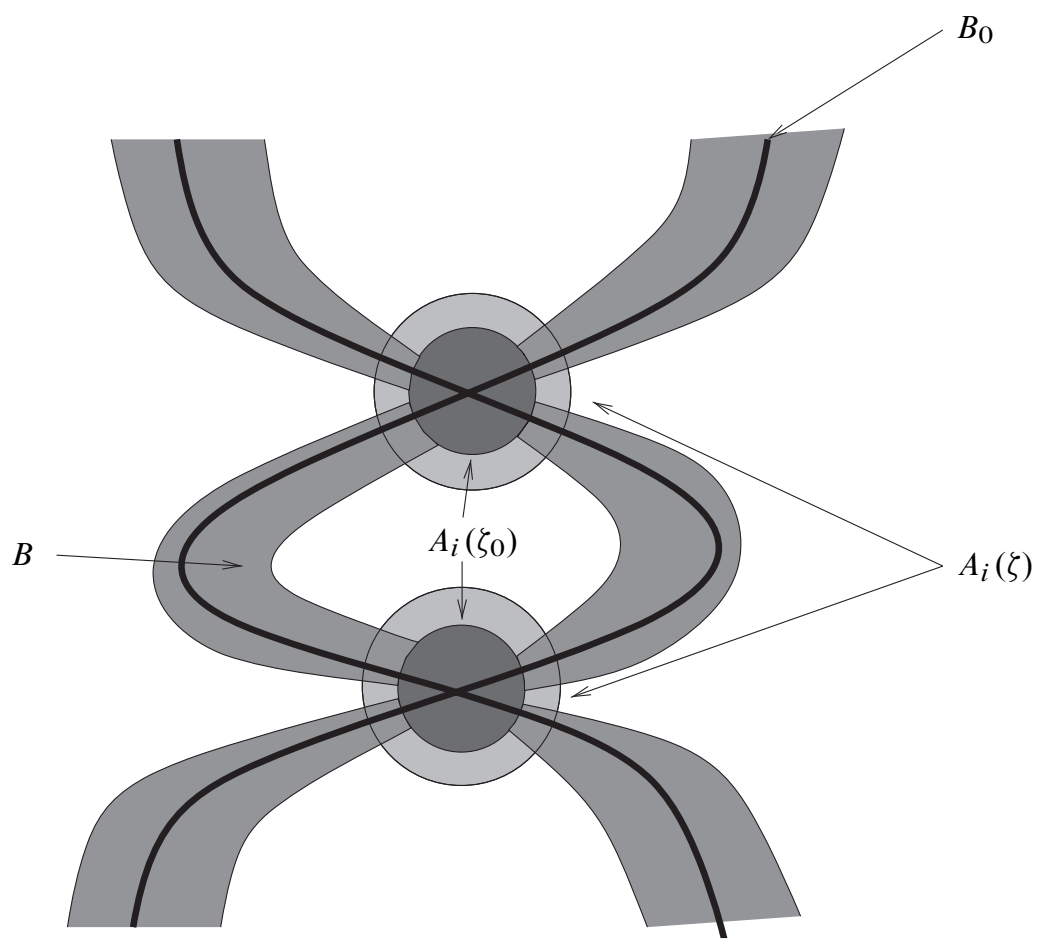

Figure 2. The decomposition of $\Sigma_{S}$.

The space $B$ is a tubular neighbourhood of $B_{0}$ in $\Sigma_{s} \backslash\left(\bigcup \dot{A}_{i}\left(\zeta_{0}\right)\right)$. The mapping $\operatorname{det}\left(H\left(f_{s}\right)\right): B \rightarrow D_{\xi}$

is a trivial fibration. Therefore there is a product structure $B \cong B_{0} \times D_{\xi}$ and the projection

$$
\rho: B \rightarrow B_{0}
$$

to the first factor induces a diffeomorphism

$$
\sigma_{u}: B_{u} \rightarrow B_{0}
$$

for any $u$.

The restriction

$$
\left.\rho \circ \pi\right|_{T_{B}}: T_{B} \rightarrow B_{0}
$$


is a locally trivial fibration with fibre a polycylinder of complex dimension $n-2$. Define

$$
\mathscr{B}:=\mathcal{M} \cap T_{B},
$$

the piece of $\mathcal{M}$ falling over $B$. Taking $T$, $\xi$ and $t_{0}$ sufficiently small we have that the restriction

$$
\left.\rho \circ \pi\right|_{\mathscr{B}}: \mathscr{B} \rightarrow B_{0}
$$

is a locally trivial fibration with fibre diffeomorphic to the Milnor fibre of the $D(1,1)$ in $\mathbb{C}^{n-1}$, and hence homotopy equivalent to $\mathbb{S}^{n-3}$ (see Lemma 4.3).

For any $\xi^{\prime}>0$ we define

$$
\begin{aligned}
U_{\xi^{\prime}} & :=\Sigma_{s} \backslash \operatorname{det}\left(H\left(f_{s}\right)\right)^{-1}\left(\dot{D}_{\xi^{\prime}}\right), \\
U_{\xi^{\prime}} & :=\pi^{-1}\left(U_{\xi^{\prime}}\right) \cap \mathcal{M},
\end{aligned}
$$

the complement of a tube around $\overline{\Sigma[1]_{s}}$ in $\Sigma_{s}$, and the piece of $\mathcal{M}$ lying over it. For $T$ and $t_{0}$ small enough, the restriction

$$
\pi \mid u_{\xi^{\prime}}: u_{\xi^{\prime}} \rightarrow U_{\xi^{\prime}}
$$

is a locally trivial fibration with fibre diffeomorphic to the Milnor fibre of the Morse singularity in $\mathbb{C}^{n-3}$, and hence homotopic to $\mathbb{S}^{n-4}$ (see Lemma 4.3).

We fix a positive $\xi_{0}$ smaller and close to $\xi$ and define

$$
\begin{aligned}
& U:=U_{\xi_{0}}, \\
& u:=U_{\xi_{0}} .
\end{aligned}
$$

The restriction

$$
\pi \mid u: U \rightarrow U
$$

is locally trivial with fibre homotopic to $\mathbb{S}^{n-4}$.

Fix a point $u$ in $\partial D_{\xi_{0}}$. Define

$$
\mathcal{B}_{u}:=\pi^{-1}\left(B_{u}\right) \cap \mathcal{M} .
$$

The mapping

$$
\left.\rho\right|_{B_{u}}: B_{u} \rightarrow B_{0}
$$

is a diffeomorphism. Hence the mapping

$$
\left(\left.\left(\left.\rho\right|_{B_{u}}\right)^{-1} \circ \rho \circ \pi\right|_{\mathscr{B}},\left.\pi\right|_{\mathcal{B}_{u}}\right):\left(\mathscr{B}, \mathscr{B}_{u}\right) \rightarrow B_{u}
$$

is a locally trivial fibration of pairs with fibre the pair $\left(\mathbb{S}^{n-3}, \mathbb{S}^{n-4}\right)$, being $\mathbb{S}^{n-4}$ embedded as an equator of $\mathbb{S}^{n-3}$. 


\section{The topology of $B_{0}$}

In this section we study the topology of $B_{0}$, which will be used later to study the homology of $\mathcal{B}$.

6.1. The fundamental group of $\boldsymbol{B}_{\mathbf{0}}$. The space $\operatorname{SM}(k)$ of symmetric matrices of size $k$ with complex coefficients is a complex vector space of dimension $k(k+1) / 2$. The smooth locally closed algebraic subset $\operatorname{SM}(k, l)$ has codimension $l(l+1) / 2$, and we have seen that its Zariski closure $\overline{\operatorname{SM}(k, l)}$ is defined by the vanishing of all $l \times l$ minors. It is easy to check that $\overline{\operatorname{SM}(k, l)}$ is far to be, in general, a complete intersection.

Define $\mathrm{MM}(k \times(k-1))$ to be the set of $(k \times(k-1))$ matrices of maximal rank.

Lemma 6.1. The fundamental group of $\operatorname{MM}(k \times(k-1))$ is trivial.

Proof. The set of matrices $k \times(k-1)$ which are not of maximal rank is an algebraic subvariety of codimension at least 2 .

The mapping

$$
\alpha_{k}: \operatorname{MM}(k \times(k-1)) \rightarrow \operatorname{SM}(k, 1)
$$

given by

$$
\alpha_{k}(M):=M M^{t}
$$

is a locally trivial fibration (by homogeneity of the action of the general linear group). Denote by $F_{k}$ the fibre over the matrix $A=\left(a_{i, j}\right)$, where $a_{i, j}=\delta_{i j}$ unless $i=j=$ $k$, in which case, $a_{k, k}=0$.

Lemma 6.2. The fiber $F_{k}$ has two connected components.

Proof. We will work by induction over $k \geq 2$. For $k=2$, it is a direct computation.

Now let us compute the fibre $F_{k}$. Consider the following matricial equation:

$$
\left(m_{i, j}\right)\left(m_{j, i}\right)=\left(a_{i, j}\right) .
$$

Let $v_{i}$ be the vector in $\mathbb{C}^{k-1}$ given by the $i$-th row of $\left(m_{i, j}\right)$. Denote by $\operatorname{Re}\left(v_{i}\right)$ and $\operatorname{Im}\left(v_{i}\right)$ its real and imaginary parts respectively.

Now the previous matricial equation becomes the following system of vector equations: if $(i, j) \neq(k, k)$ then

$$
\begin{gathered}
\operatorname{Re}\left(v_{i}\right) \cdot \operatorname{Re}\left(v_{j}\right)=\delta_{i j}+\operatorname{Im}\left(v_{i}\right) \cdot \operatorname{Im}\left(v_{j}\right), \\
\operatorname{Re}\left(v_{i}\right) \cdot \operatorname{Im}\left(v_{j}\right)=0
\end{gathered}
$$

and

$$
\operatorname{Re}\left(v_{k}\right) \cdot \operatorname{Re}\left(v_{k}\right)=\operatorname{Im}\left(v_{k}\right) \cdot \operatorname{Im}\left(v_{k}\right)
$$




$$
\operatorname{Re}\left(v_{k}\right) \cdot \operatorname{Im}\left(v_{k}\right)=0,
$$

where $v \cdot w$ denotes the standard scalar product in $\mathbb{R}^{k-1}$.

Consider the projection $\operatorname{MM}(k \times(k-1)) \subset\left(\mathbb{C}^{k-1}\right)^{k} \rightarrow \mathbb{C}^{k-1}$ to the first row. Let $B_{k}$ be the image of $F_{k}$ under this projection. Using the homogeneity of the action of the complex orthogonal group, it is easy to check that the restriction

$$
\tau_{k}: F_{k} \rightarrow B_{k}
$$

is a locally trivial fibration.

Obviously $B_{k}$ is the set of vectors $v_{1}$ satisfying the above system of equations for $i=j=1$. The vector $v_{1}$ belongs to $B_{k}$ if and only if $\left\|\operatorname{Re}\left(v_{1}\right)\right\|^{2}$ is one unit longer than $\left\|\operatorname{Im}\left(v_{1}\right)\right\|^{2}$ and both vectors are orthogonal. That is, the vector $\operatorname{Re}\left(v_{1}\right)$ can be anywhere except in the interior of the unit sphere in $\mathbb{R}^{k-1}$. If $\left\|\operatorname{Re}\left(v_{1}\right)\right\|^{2}$ equals 1 then the vector $\operatorname{Im}\left(v_{1}\right)$ is zero. In any other case, the vector $\operatorname{Im}\left(v_{1}\right)$ lies in the $(k-2)$-sphere of radius $\sqrt{1-\left\|\operatorname{Re}\left(v_{1}\right)\right\|^{2}}$ in the hyperplane orthogonal to $\operatorname{Re}\left(v_{1}\right)$. It is easy to show that $B_{k}$ admits the unit sphere in $\mathbb{R}^{k-1}$ embedded in the real part of $\mathbb{C}^{k-1}$ as a deformation retract.

The fiber $\tau_{k}^{-1}((1,0, \ldots, 0))$ is formed by the $(k-1)$-tuples $\left(v_{2}, \ldots, v_{k}\right)$ of vectors that satisfy the previous system of vector equations, and lie inside the subspace of vectors with a zero in the first coordinate. This is equal to the fiber $F_{k-1}$ of $\alpha_{k-1}$ over $A^{\prime}$ where $A^{\prime}$ is the result of deleting the first row and the first column in $A$.

We have constructed a fibration of $F_{k}$ over a space with the homotopy type of $S^{k-2}$ whose fibre is $F_{k-1}$. If $F_{k-1}$ has two connected components and $k \geq 4$, the homotopy exact sequence of the fibrations gives the result. For $k=3$ we have to check that the monodromy of the fibration does not interchange the two connected components of $F_{2}$, but this is direct computation.

Proposition 6.3. The fundamental group of $\operatorname{SM}(k, 1)$ is isomorphic to $\mathbb{Z}_{2}$.

Proof. This is just the homotopy exact sequence of the fibration $\alpha_{k}$, together with Lemmas 6.1 and 6.2.

Proposition 6.4. The fundamental group of $B_{0}$ is isomorphic to $\mathbb{Z}_{2}$.

Proof. The unfolding

$$
f_{s}=\left(g_{1}-s_{1}, \ldots, g_{n-3}-s_{n-3}\right)\left(m_{i, j}+s_{i, j}\right)\left(g_{1}-s_{1}, \ldots, g_{n-3}-s_{n-3}\right)^{t},
$$

with $s \in \mathbb{C}^{n-3} \times \operatorname{SM}(n-3)$ can be obtained by pullback from the unfoldings (1) and (6) of $f$ that we considered in Section 2 both in the corank $(H(f)(O))=2$ and corank $(H(f)(O)) \neq 2$ cases. In both cases a generic parameter

$$
s \in \mathbb{C}^{n-3} \times \operatorname{SM}(n-3)
$$


maps to a parameter outside the discriminant $\Delta$. Thus we can use this unfolding in order to compute the topology of $B_{0}$.

The mapping

$$
\alpha: \mathbb{C}^{n} \times \operatorname{SM}(n-3) \rightarrow \operatorname{SM}(n-3)
$$

defined by $\alpha\left(x,\left(s_{i, j}\right)\right):=\left(m_{i, j}(x)+s_{i, j}\right)$ is obviously a submersion wherever it is defined. Define

$$
\left.\mathrm{Z}_{i}:=\alpha^{-1}(\overline{\operatorname{SM}(n-3, i})\right)
$$

Since $\overline{\operatorname{SM}(n-3, i)}$ is a cone for any $i$, and the fundamental group of $\operatorname{SM}(n-3,1)$ is isomorphic to $\mathbb{Z}_{2}$, we have that the local fundamental group of the germ $\operatorname{SM}(n-3,1)$ at the origin is $\mathbb{Z}_{2}$. Since the mapping $\alpha$ is a submersion, the local fundamental group of $\left(Z_{1} \backslash Z_{2}\right)$ at the origin is $\mathbb{Z}_{2}$.

Fix a positive $\epsilon$ and a generic

$$
s^{0}=\left(s_{1}^{0}, \ldots, s_{n-3}^{0},\left(s_{i, j}^{0}\right)_{1 \leq i \leq j \leq n-3}\right) \in \mathbb{C}^{n-3} \times \operatorname{SM}(n-3)
$$

sufficiently close to the origin. Consider set of functions $\left\{g_{i}\right\}_{i=1}^{n-3} \cup\left\{s_{i, j}\right\}_{1 \leq i \leq j \leq n-3}$ in $\mathcal{O}_{\mathbb{C}^{n} \times \operatorname{SM}(n-3)}$. Applying Hamm-Lê Theorem (Main Theorem in [8], II.1.4) repeatedly for the above set of functions, and using the relative homotopy exact sequence we get that the fundamental group of

$$
B_{\epsilon} \cap\left(\mathcal{Z}_{1} \backslash \mathcal{Z}_{2}\right) \cap \bigcap_{i=1}^{n-3} V\left(g_{i}-s_{i}^{0}\right) \cap \bigcap_{1 \leq i \leq j \leq n-3} V\left(s_{i, j}-s_{i, j}^{0}\right)
$$

is isomorphic to $\mathbb{Z}_{2}$. But it is clear that the above space is homotopic to $B_{0}$.

6.2. Homology of $\boldsymbol{B}_{\mathbf{0}}$. We will now compute the homology of $B_{0}$, which coincides with the homology of $B$. Given the function

$$
\operatorname{det}\left(H\left(f_{s}\right)\right): \Sigma_{s} \rightarrow \mathbb{C}
$$

we use the Mayer-Vietoris sequence of the $\operatorname{decomposition}$ of $\operatorname{det}\left(H\left(f_{S}\right)\right)^{-1}\left(D_{\xi}\right)$ as the union of $\bigcup A_{i}(\zeta)$ and $B$ given in Section 5.

The space $\operatorname{det}\left(H\left(f_{s}\right)\right)^{-1}\left(D_{\xi}\right)$ is homotopy equivalent to $\operatorname{det}\left(H\left(f_{s}\right)\right)^{-1}(0)$, which is homotopic to a bouquet of $\left(\mu_{1}-a\right)$ 2-spheres (see Definition 2.5). This is because $\operatorname{det}\left(H\left(f_{s}\right)\right)^{-1}(0)$ is a deformation of $\operatorname{det}(H(f))^{-1}(0)$, which is an i.c.i.s. with Milnor number $\mu_{1}$, and $\operatorname{det}\left(H\left(f_{s}\right)\right)^{-1}(0)$ has only a Morse-points as singularities.

On the other hand, the intersection of each space $A_{i}(\zeta)$ with $B$ is the link $\mathbb{R P}^{3}$ of a Morse type singularity, and the spaces $A_{i}(\zeta)$ are contractible.

Summarising, we have the following:

- $H_{3}\left(\bigcup A_{i}(\zeta) \cap B ; \mathbb{Z}\right) \cong \mathbb{Z}^{a}$,

- $H_{1}\left(\bigcup A_{i}(\zeta) \cap B ; \mathbb{Z}\right) \cong \mathbb{Z}_{2}^{a}$, 
- $H_{i}\left(\bigcup A_{i}(\zeta) \cap B ; \mathbb{Z}\right) \cong 0$ for $i \notin\{0,1,3\}$,

- $H_{2}\left(\operatorname{det}\left(H\left(f_{s}\right)\right)^{-1}\left(D_{\xi}\right) ; \mathbb{Z}\right) \cong \mathbb{Z}^{\mu_{1}-\mathfrak{a}}$,

- $H_{i}\left(\operatorname{det}\left(H\left(f_{s}\right)\right)^{-1}\left(D_{\xi}\right) ; \mathbb{Z}\right) \cong 0$ for $i \notin\{0,2\}$,

- $H_{1}(B ; \mathbb{Z}) \cong \mathbb{Z}_{2}$.

These data allow us to compute the following Mayer-Vietoris sequence:

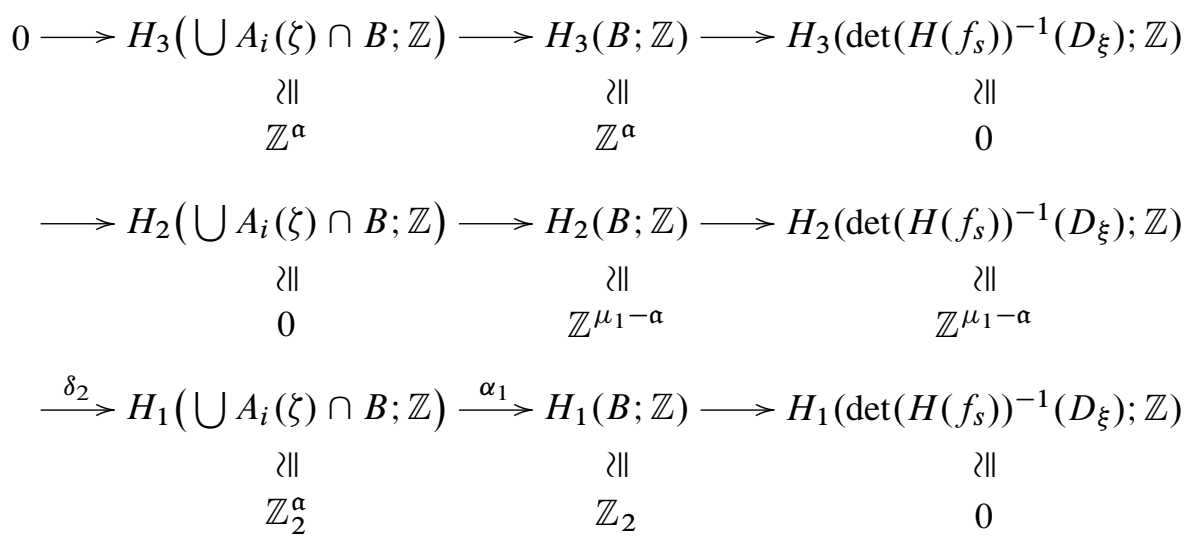

Remark 6.5. The restriction of the mapping $\alpha_{1}$ to $H_{1}\left(A_{i}(\zeta) \cap B ; \mathbb{Z}\right)$ is an isomorphism onto $H_{1}(B ; \mathbb{Z})$ for any $i$.

Proof. The space $B$ is homotopy equivalent to $B_{0}$. In Proposition 6.4 it is proved that the fundamental group of $B_{0}$ is isomorphic to $\mathbb{Z}_{2}$, and hence the same holds for its first homology group.

The group $H_{1}\left(\bigcup A_{i}(\zeta) \cap B ; \mathbb{Z}\right)$ is a direct sum of the groups $H_{1}\left(A_{i}(\zeta) \cap B ; \mathbb{Z}\right)$ for $i$ varying. Each of these groups is isomorphic to $\mathbb{Z}_{2}$, and is generated by a homology class $\left[\gamma_{i}\right]$. Since the exact sequence above shows that $\alpha_{1}$ is surjective, at least one $\left[\gamma_{i}\right]$ has non-zero image by $\alpha_{1}$ in $H_{1}(B ; \mathbb{Z})$.

Lemma 2.4 implies shows that there are isomorphism of $B_{0}$ interchanging each two classes $\left[\gamma_{i}\right]$. This ends the proof.

The homology of $B$ with coefficients in $\mathbb{Z}_{2}$ can be computed either in a similar way than the integral one, or by using the universal coefficient theorem. We obtain

- $H_{4}\left(B ; \mathbb{Z}_{2}\right)=0$,

- $H_{3}\left(B ; \mathbb{Z}_{2}\right)=\mathbb{Z}_{2}^{a}$,

- $H_{2}\left(B ; \mathbb{Z}_{2}\right)=\mathbb{Z}_{2} \oplus \mathbb{Z}_{2}^{\mu_{1}-\mathfrak{a}}$,

- $H_{1}\left(B ; \mathbb{Z}_{2}\right)=\mathbb{Z}_{2}$. 
Remark 6.6. Note that the generators of $H_{2}\left(\operatorname{det}\left(H\left(f_{s}\right)\right)^{-1}\left(D_{\xi}\right) ; \mathbb{Z}\right)$ can be interpreted as follows. The Milnor fibre of $\operatorname{det}\left(H\left(f_{0}\right)\right)^{-1}(0)$ has $\mu_{1} 2$-spheres as generators of its homology. Out of these spheres there are a of them which correspond to the vanishing cycles of the a Morse points of $\operatorname{det}\left(H\left(f_{S}\right)\right)^{-1}(0)$. The space $\operatorname{det}\left(H\left(f_{s}\right)\right)^{-1}\left(D_{\xi}\right)$ is homotopic to $\operatorname{det}\left(H\left(f_{s}\right)\right)^{-1}(0)$, which in turn is homotopic to the result of collapsing these a spheres in the Milnor fibre of $\operatorname{det}\left(H\left(f_{0}\right)\right)^{-1}(0)$. The remaining spheres give rise to the $\mu_{1}-$ a generators of $H_{2}\left(\operatorname{det}\left(H\left(f_{s}\right)\right)^{-1}\left(D_{\xi}\right) ; \mathbb{Z}\right)$.

\section{Homology of $\left(\mathscr{B}, \mathscr{B}_{u}\right)$}

In this section we will compute the homology of the spaces $\mathcal{B}$ and $\mathscr{B}_{u}$, and also the one of the pair $\left(\mathscr{B}, \mathscr{B}_{u}\right)$. We will do so by studying their fibrations over $B_{u}$. This way we will obtain the homology with integer coefficients of $\mathcal{B}_{u}$ and $\left(\mathscr{B}, \mathscr{B}_{u}\right)$; but for $\mathcal{B}$ we can only compute it with coefficients in $\mathbb{Z}_{2}$, because the associated fibration is not orientable. At the end of the section we will use these three homologies, together with the universal coefficients theorem to recover the long exact sequence of the pair with integer coefficients, and thus obtain the integer homology of $\mathcal{B}$.

There are several sphere fibrations involved in the computation of the homology of the Milnor fibre, and we will need to deal with the corresponding Gysin sequences. These are greatly simplified if we are in the case $n \geq 8$. The homology of the Milnor fibre can be always deduced (by suspension) from the homology of the Milnor fibre of a function $f:\left(\mathbb{C}^{n}, O\right) \rightarrow \mathbb{C}$ with $n \geq 8$. We will assume in $n \geq 8$ whenever is needed.

Consider the fibration $\mathscr{B}_{u} \rightarrow B_{u}$. As we have seen previously, it is a fibration, with fiber homotopically equivalent to $\mathbb{S}^{n-4}$. This fibration can be extended to $h_{s}^{-1}(u)$, which is simply connected, and hence, the fibration is orientable. Its Gysin exact sequence leads to the isomorphisms

$$
H_{i}\left(B_{u} ; \mathbb{Z}\right) \cong H_{n-4+i}\left(\mathcal{B}_{u} ; \mathbb{Z}\right), \quad H_{i}\left(\mathcal{B}_{u} ; \mathbb{Z}\right) \cong H_{i}\left(B_{u} ; \mathbb{Z}\right)
$$

for $i=0,1,2,3$. The rest of the homology groups of $\mathcal{B}_{u}$ vanish.

Consider the projection $\mathcal{B} \rightarrow B_{u}$. As we have seen before, it is a fibration with fibre homotopically equivalent to $\mathbb{S}^{n-3}$, and the monodromy reverses the orientation. Since the fibration is not orientable, we can only consider its Gysin sequence with coefficients in $\mathbb{Z}_{2}$, which gives the following isomorphisms:

$$
H_{i}\left(B_{u} ; \mathbb{Z}_{2}\right) \cong H_{n-3+i}\left(\mathcal{B} ; \mathbb{Z}_{2}\right), \quad H_{i}\left(\mathscr{B} ; \mathbb{Z}_{2}\right) \cong H_{i}\left(B_{u} ; \mathbb{Z}_{2}\right)
$$

for $i=0,1,2,3$. The rest of the homology groups of $\mathcal{B}$ with coefficients in $\mathbb{Z}_{2}$ vanish.

The fibration of pairs $\left(\mathcal{B}, \mathscr{B}_{u}\right) \rightarrow B_{u}$ has as fibre the pair $\left(\mathbb{S}^{n-3}, \mathbb{S}^{n-4}\right)$ with $\mathbb{S}^{n-4}$ embedded as the equator of $\mathbb{S}^{n-3}$. Its monodromy acts trivially on $\mathbb{S}^{n-4}$ and reverses the hemispheres of $\mathbb{S}^{n-3}$ along the only non-trivial class of $\pi_{1}\left(\mathscr{B}_{u}\right) \cong \mathbb{Z}^{2}$. 
In order to compute the homology of the pair $\left(\mathscr{B}, \mathscr{B}_{u}\right)$ we can simultaneously thicken the equator $\mathbb{S}^{n-4}$ of each fibre to a small collar $\mathbb{S}^{n-4} \times[-\eta, \eta]$ in $\mathbb{S}^{n-3}$. By excision we can remove fibrewise the interior of the collar. We obtain a fibration over $B_{u}$ with fibre two $(n-3)$-disks relative to their boundary, such that the monodromy interchanges them.

Since $\pi_{1}\left(B_{u}\right) \cong \mathbb{Z}_{2}$, its universal cover

$$
\sigma: \widetilde{B}_{u} \rightarrow B_{u}
$$

is the only connected double cover. The fibration of pairs is then homologically equivalent to the composition of an orientable fibration

$$
\varphi: y \rightarrow \widetilde{B}_{u}
$$

of $(n-3)$-spheres over $\widetilde{B}_{u}$ with the covering map $\sigma$. The Gysin sequence of the fibration $\varphi$ gives

$$
H_{k}\left(\mathscr{B}, \mathscr{B}_{u} ; \mathbb{Z}\right) \cong H_{k}(\mathcal{Y} ; \mathbb{Z}) \cong H_{k-(n-3)}\left(\widetilde{B}_{u} ; \mathbb{Z}\right)
$$

if $k \geq n-3$ and zero otherwise.

Denote by $\mathcal{V}$ the double cover of $\overline{\Sigma[1]_{s}}$ branched over its a singular points. The space $\widetilde{B}_{u}$ is homotopically equivalent to $\mathcal{V}$ minus the preimage by the covering map of these a points. The space $\mathcal{V}$ is a 2-dimensional Stein space (for being a branched cover of the 2-dimensional Stein space $\overline{\Sigma[1]_{s}}$ ), and hence it has the homotopy type of a 2-dimensional CW-complex. Therefore, $H_{2}(\mathcal{V} ; \mathbb{Z})$ is free and $H_{3}(\mathcal{V} ; \mathbb{Z})$ vanishes. Since the singularities of $B_{0}$ are of Morse type, and the 2-dimensional Morse singularity is the quotient of $\mathbb{C}^{2}$ by the action of the group of two elements, the space $\mathcal{V}$ is smooth. Hence $\widetilde{B}_{u}$ is the result of deleting from $\mathcal{V}$ small balls around the a preimages by the double cover of the singular points of $\overline{\Sigma[1]_{s}}$. Using the MayerVietoris sequence we see that such deletion leaves unchanged the homology except in dimension 3, where we obtain a copy of $\mathbb{Z}$ for each deleted point. Summarising, we get that

- $H_{3}\left(\widetilde{B}_{u} ; \mathbb{Z}\right)=\mathbb{Z}^{a}$

- $H_{2}\left(\widetilde{B}_{u} ; \mathbb{Z}\right)=\mathbb{Z}^{k}$ for a certain $k$

- $H_{1}\left(\widetilde{B}_{u} ; \mathbb{Z}\right)=0$, since it is the universal cover of $B_{u}$

- $H_{0}\left(\widetilde{B}_{u} ; \mathbb{Z}\right)=\mathbb{Z}$, for it is connected.

Since the Euler characteristic of $\widetilde{B}_{u}$ is twice the one of $B_{u}, k$ must be equal to $2 \mu_{1}-3 a+1$.

It is is easy to check that the following diagram is commutative:

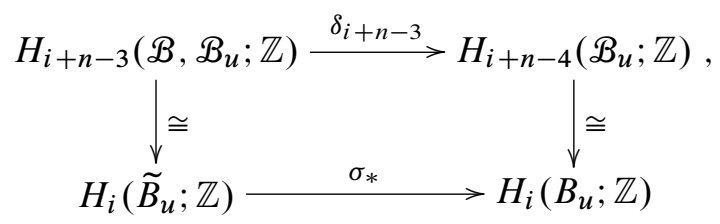


for any $i$, where $\delta_{i+n-3}$ is the connecting homomorphism of the long exact sequence of the pair $\left(\mathscr{B}, \mathscr{B}_{u}\right)$, the mapping $\sigma: \widetilde{B}_{u} \rightarrow B_{u}$ is the covering map and the vertical arrows are the isomorphisms coming from the Gysin sequences.

Notice that the generators of $H_{3}\left(\widetilde{B}_{u} ; \mathbb{Z}\right)$ are 3 -spheres bounding balls in $\widetilde{B}_{0}$ around the inverse image of the singularities of $B_{0}$. The generators of $H_{3}\left(B_{u} ; \mathbb{Z}\right)$ are precisely the classes $\left[A_{i}(\eta) \cap B_{u}\right]$. Each of them is diffeomorphic to $\mathbb{R P}^{3}$ and doubly covered by one of the 3 -spheres. This shows that

$$
\pi_{*}: H_{3}\left(\widetilde{B}_{u} ; \mathbb{Z}\right) \rightarrow H_{3}\left(B_{u} ; \mathbb{Z}\right),
$$

and hence also $\delta_{n}$, is multiplication by 2 .

For being $\sigma$ a covering there is a well defined pull-back mapping in homology

$$
\sigma^{*}: H_{i}\left(B_{u} ; \mathbb{Z}\right) \rightarrow H_{i}\left(\widetilde{B}_{u} ; \mathbb{Z}\right)
$$

It is clear that the map $\sigma_{*} \sigma^{*}: H_{i}\left(B_{u} ; \mathbb{Z}\right) \rightarrow H_{i}\left(B_{u} ; \mathbb{Z}\right)$ is multiplication by 2 (the degree of the covering). This, together with the previous commutative diagram, implies that $2 H_{i-1}\left(\mathcal{B}_{u} ; \mathbb{Z}\right)$ is always in the image of $\delta_{i}$ for any $i$. In view of this and of the long exact sequence of the pair $\left(\mathscr{B}, \mathscr{B}_{u}\right)$ we obtain that $H_{n-2}(\mathscr{B}, \mathbb{Z})$ can not have $p$-torsion for $p \neq 2$.

By the above diagram and the connectedness of $\widetilde{B}_{u}$ we have that $\delta_{n-3}$ is an isomorphism.

These facts, together with the previous computations of $H_{\bullet}\left(\mathcal{B}_{u} ; \mathbb{Z}\right), H_{\bullet}\left(\mathscr{B} ; \mathbb{Z}_{2}\right)$ and $H_{\bullet}\left(\mathscr{B}, \mathscr{B}_{u} ; \mathbb{Z}\right)$, plus the universal coefficients theorem allow us to completely determine the long integral homology exact sequence of the pair $\left(\mathscr{B}, \mathscr{B}_{u}\right)$, (equation (17)).

The non-zero lower homology groups are isomorphic to those of $\mathscr{B}_{u}$, which coincide with those of $B$.

\section{Homology of $x$}

This section is devoted to the computation of the homology of $\mathcal{X}$. In a first step we will compute it with coefficients in $\mathbb{Z}_{2}$ using the Mayer-Vietoris sequence. Then we will lift it to the integers using the universal coefficients theorem.

Let $\mathcal{X}$ be the union of $\bigcup_{i=1}^{a} \mathcal{A}_{i}$ and $\mathcal{B}$. We will now consider the MayerVietoris sequence of this union with coefficients in $\mathbb{Z}_{2}$. To do so, we need to compute the groups $H_{\bullet}\left(\mathcal{A}_{i} ; \mathbb{Z}_{2}\right)$ and $H_{\bullet}\left(\mathcal{A}_{i} \cap \mathcal{B} ; \mathbb{Z}_{2}\right)$, since $H_{\bullet}\left(\mathcal{B} ; \mathbb{Z}_{2}\right)$ has already been computed.

The space $\mathcal{A}_{i}$ is the Milnor fiber of the singularity $D(3,2)$, and hence, it has the homotopy type of the sphere $\mathbb{S}^{n-2}$.

To study the homology of $\mathcal{A}_{i} \cap \mathcal{B}$, we can use the Gysin sequence of the fibration

$$
\pi: \mathcal{A}_{i} \cap \mathcal{B} \rightarrow A_{i} \cap B \simeq \partial\left(A_{i} \cap \operatorname{det}\left(H\left(f_{s}\right)\right)^{-1}(0)\right) \cong \mathbb{R P}^{3},
$$




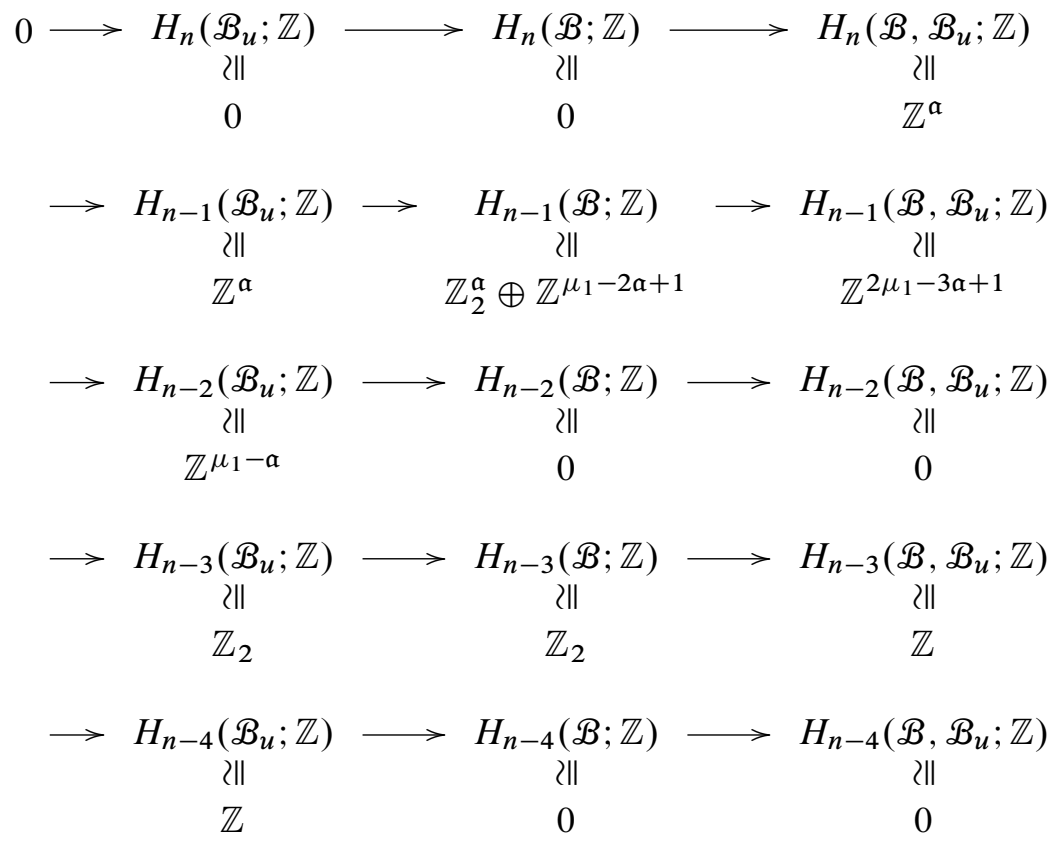

with fibre $\mathbb{S}^{n-3}$. The groups $H_{i}\left(\mathbb{R} \mathbb{P}^{3} ; \mathbb{Z}_{2}\right)$ are $\mathbb{Z}_{2}$ for $i=0,1,2,3$, and zero otherwise. We obtain that

$$
H_{i}\left(\mathcal{A}_{i} \cap \mathcal{B}, \mathbb{Z}_{2}\right)= \begin{cases}\mathbb{Z}_{2} & \text { for } i=0,1,2,3, n-3, n-2, n-1, n, \\ 0 & \text { otherwise. }\end{cases}
$$

To study the maps $\iota_{k}: \bigoplus_{i} H_{k}\left(\mathcal{A}_{i} \cap \mathscr{B} ; \mathbb{Z}_{2}\right) \rightarrow H_{k}\left(\mathscr{B} ; \mathbb{Z}_{2}\right)$ induced by inclusion, we will see them as the Gysin lift of the maps $\bigoplus_{i} H_{j}\left(A_{i} \cap B ; \mathbb{Z}_{2}\right) \rightarrow H_{j}\left(B ; \mathbb{Z}_{2}\right)$ for $j=k$ or $j=k-n+3$. Using the version of the Mayer Vietoris sequence (14) with coefficients in $\mathbb{Z}_{2}$, we get easily that

- $\iota_{n}$ and $\iota_{3}$ are isomorphisms,

- $\iota_{n-1}$ is a monomorphism,

- $\iota_{n-2}$ and $\iota_{n-3}$ are epimorphisms,

- $\iota_{1}$ is an epimorphism,

- $\iota_{2}$ is a monomorphism.

We need also the following lemma, whose proof we postpone to page 290:

Lemma 8.1. The map $\iota_{2}: H_{n-2}\left(\mathcal{A}_{i} \cap \mathscr{B} ; \mathbb{Z}_{2}\right) \rightarrow H_{n-2}\left(\mathcal{A}_{i} ; \mathbb{Z}_{2}\right)$ induced by inclusion is an isomorphism. 
With all these facts, we can compute the corresponding Mayer-Vietoris sequence (equation (18)).

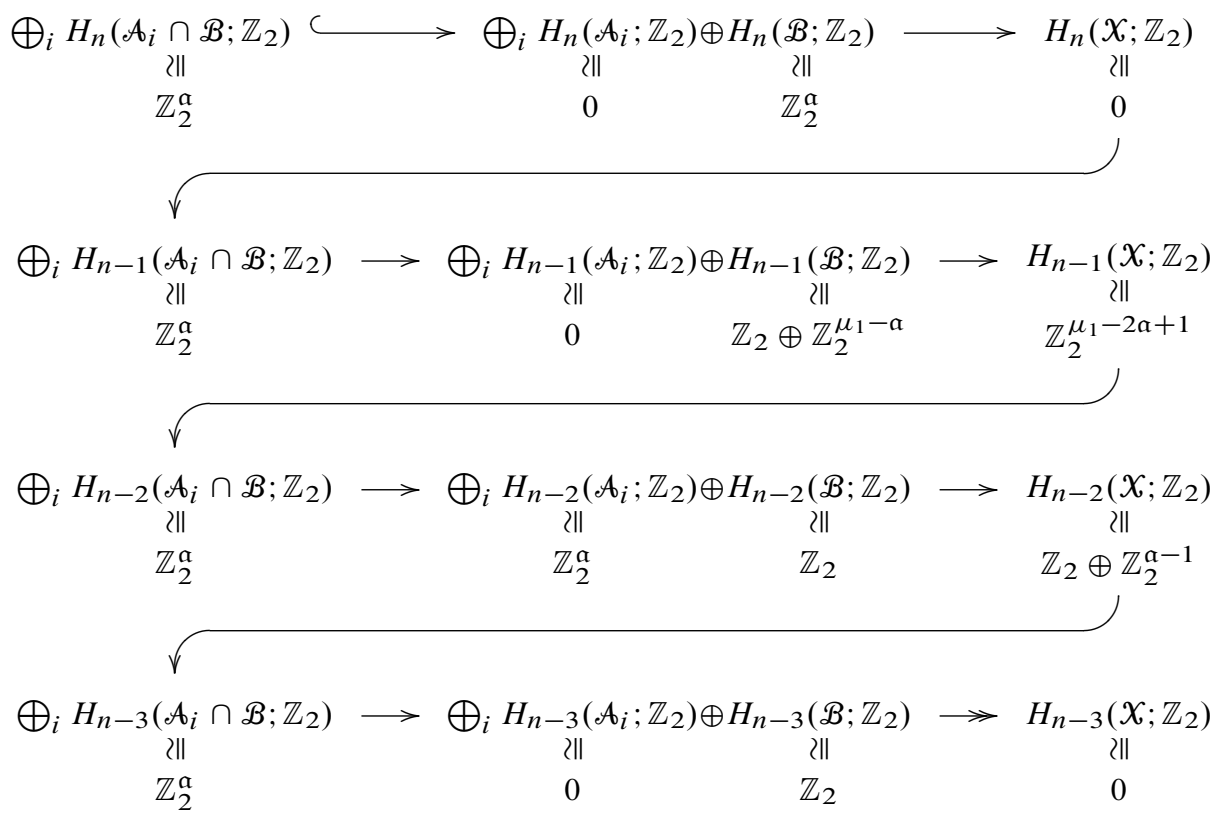

We omit the lower part of the sequence. The non-vanishing remaining homology groups of $\mathcal{X}$ are

$$
H_{2}\left(X ; \mathbb{Z}_{2}\right) \cong \mathbb{Z}_{2}^{\mu_{1}-\mathfrak{a}}, \quad H_{0}\left(\mathcal{X} ; \mathbb{Z}_{2}\right) \cong \mathbb{Z}_{2}
$$

8.1. A basis of $\boldsymbol{H}_{\boldsymbol{n}-2}\left(\boldsymbol{X} ; \mathbb{Z}_{2}\right)$. Fix a base point $x_{1} \in A_{1}(\zeta) \cap B_{u}$. Choose paths $\gamma_{i}:[0,1] \rightarrow B_{u}$ such that $\gamma_{1}$ is a generator of the fundamental group of $A_{1}(\zeta) \cap B_{u}$, and $\gamma_{i}$ connects $x_{1}$ with some point $x_{i} \in A_{i}(\zeta) \cap B_{u}$. We choose chains $G_{i} \subset$ $\mathcal{B}$ such that the natural projection $\left.\pi\right|_{G_{i}}$ is a locally trivial fibration over $\gamma_{i}$ with fibre diffeomorphic to a $\mathbb{S}^{n-3}$ generating the homology of the corresponding fibre of $\left.\left(\left.\rho\right|_{B_{u}}\right)^{-1} \circ \rho \circ \pi\right|_{\mathcal{B}}$. Since $\gamma_{1}$ is closed, the chain $G_{1}$ is closed with coefficients in $\mathbb{Z}_{2}$. For each $i$, we choose an $(n-2)$-sphere generating $H_{n-2}\left(\mathcal{A}_{i} ; \mathbb{Z}\right)$. Take a hemisphere $K_{i}$ of such sphere; its boundary $\partial K_{i}$ is an $(n-3)$-sphere in $\mathcal{A}_{i}$. The boundary $\partial G_{i}$ consists of two $(n-3)$-spheres $L_{1}$ and $L_{i}$, being $L_{i}$ contained in $\mathcal{A}_{i}$. Since $\mathcal{A}_{i}$ is homotopic to $\mathbb{S}^{n-2}$ there exists a chain

$$
W_{i}:[0,1] \times \mathbb{S}^{n-3} \rightarrow \mathcal{A}_{i}
$$

such that $\partial W_{i}=\partial K_{i}+L_{i}$. 
The generators of $H_{n-2}\left(X ; \mathbb{Z}_{2}\right)$ are represented by the $\mathbb{Z}_{2}$-closed chains $Z_{1}:=$ $G_{1}$ and $Z_{i}:=K_{1}+W_{1}+G_{i}+W_{i}+K_{i}$. Notice that since the coefficients are in $\mathbb{Z}_{2}$ we have $K_{1}+W_{1}+C_{1}+W_{1}+K_{1}=G_{1}$, and so the way of defining the generators is the same for all indices. To check that these are really generators we observe that $Z_{2}, \ldots, Z_{a}$ are sent by the connecting homomorphism of the MayerVietoris sequence (18) to the kernel of the first mapping of the $(n-3)$ rd row, and that $Z_{1}$ generates the cokernel of the first mapping of the $(n-2)$ nd row.

Lemma 8.2. Let $\gamma_{i}^{\prime}:[0,1] \rightarrow B_{u}$ be any other path joining $x_{1}^{\prime}$ and $x_{i}^{\prime}$, being $x_{1}^{\prime}$ and $x_{i}^{\prime}$ points in $A_{1}(\zeta) \cap B$ and $A_{i}(\zeta) \cap B$ respectively. As above we can associate with $\gamma_{i}^{\prime}$ an element $\left[Z_{i}^{\prime}\right] \in H_{n-2}\left(X ; \mathbb{Z}_{2}\right)$. We have the equality

$$
\left[Z_{i}^{\prime}\right]=\left[Z_{i}\right]+m\left[Z_{1}\right]
$$

for a certain $m \in \mathbb{Z}_{2}$.

Proof. Let $\alpha_{j}$ be a path joining $x_{j}$ and $x_{j}^{\prime}$ for $j=1, i$. The product of paths $\gamma_{i} \cdot \alpha_{i} \cdot\left(\gamma_{i}^{\prime}\right)^{-1} \cdot\left(\alpha_{1}\right)^{-1}$ is a loop based in $x_{1}$. Since the fundamental group $\pi_{1}\left(B_{u}, x_{1}\right)$ is isomorphic to $\mathbb{Z}_{2}$ and generated by $\gamma_{1}$, the loop $\gamma_{i} \cdot \alpha_{i} \cdot\left(\gamma_{i}^{\prime}\right)^{-1} \cdot\left(\alpha_{1}\right)^{-1}$ is homotopic to $\left(\gamma_{1}\right)^{m}$ for a certain $m$. After this, the above equality follows easily from the construction of the chains $Z_{i}$.

8.2. A system of generators of $\boldsymbol{H}_{\boldsymbol{n}-2}(\boldsymbol{X} ; \mathbb{Z})$. To lift the computation to coefficients in $\mathbb{Z}$, we need to compute the integer homology of $\mathcal{A}_{i} \cap \mathscr{B}$. We can do so by computing the long exact sequence of the pair $\left(\mathcal{A}_{i} \cap \mathscr{B}, \mathcal{A}_{i} \cap \mathscr{B}_{u}\right)$ using the same arguments used to compute the long exact sequence of the pair $\left(\mathscr{B}, \mathscr{B}_{u}\right)$. We obtain:

$$
\begin{aligned}
H_{n-1}\left(\mathcal{A}_{i} \cap \mathcal{B} ; \mathbb{Z}\right) & \cong \mathbb{Z}_{2}, \\
H_{n-3}\left(\mathcal{A}_{i} \cap \mathcal{B} ; \mathbb{Z}\right) & \cong \mathbb{Z}_{2}, \\
H_{3}\left(\mathcal{A}_{i} \cap \mathscr{B} ; \mathbb{Z}\right) & \cong \mathbb{Z}, \\
H_{1}\left(\mathcal{A}_{i} \cap \mathscr{B} ; \mathbb{Z}\right) & \cong \mathbb{Z}_{2}, \\
H_{0}\left(\mathcal{A}_{i} \cap \mathcal{B} ; \mathbb{Z}\right) & \cong \mathbb{Z},
\end{aligned}
$$

and zero otherwise.

With these data, and the universal coefficients theorem, we can compute the Mayer-Vietoris sequence (18) with coefficients in $\mathbb{Z}$, which is shown in equation (19).

The non-zero lower homology groups are isomorphic to those of $\Sigma[1]_{s}$.

We give a system of generators of $H_{n-2}(X ; \mathbb{Z})$. For any $i$ choose an $(n-2)$-sphere $S_{i}$ in $\mathcal{A}_{i}$ generating $H_{n-2}\left(\mathcal{A}_{i} ; \mathbb{Z}\right)$. Choosing the orientations of the summands of $Z_{i}$ appropriately it turns out that we have a $\mathbb{Z}$-closed chain. It is clear that $\left[Z_{2}\right], \ldots,\left[Z_{a}\right]$ generate the kernel of the first homomorphism of the $(n-3)$ rd row of the MayerVietoris sequence. The image of the second morphism of the $(n-2)$ nd row is obviously generated by the $(n-2)$-spheres $S_{i}$. 


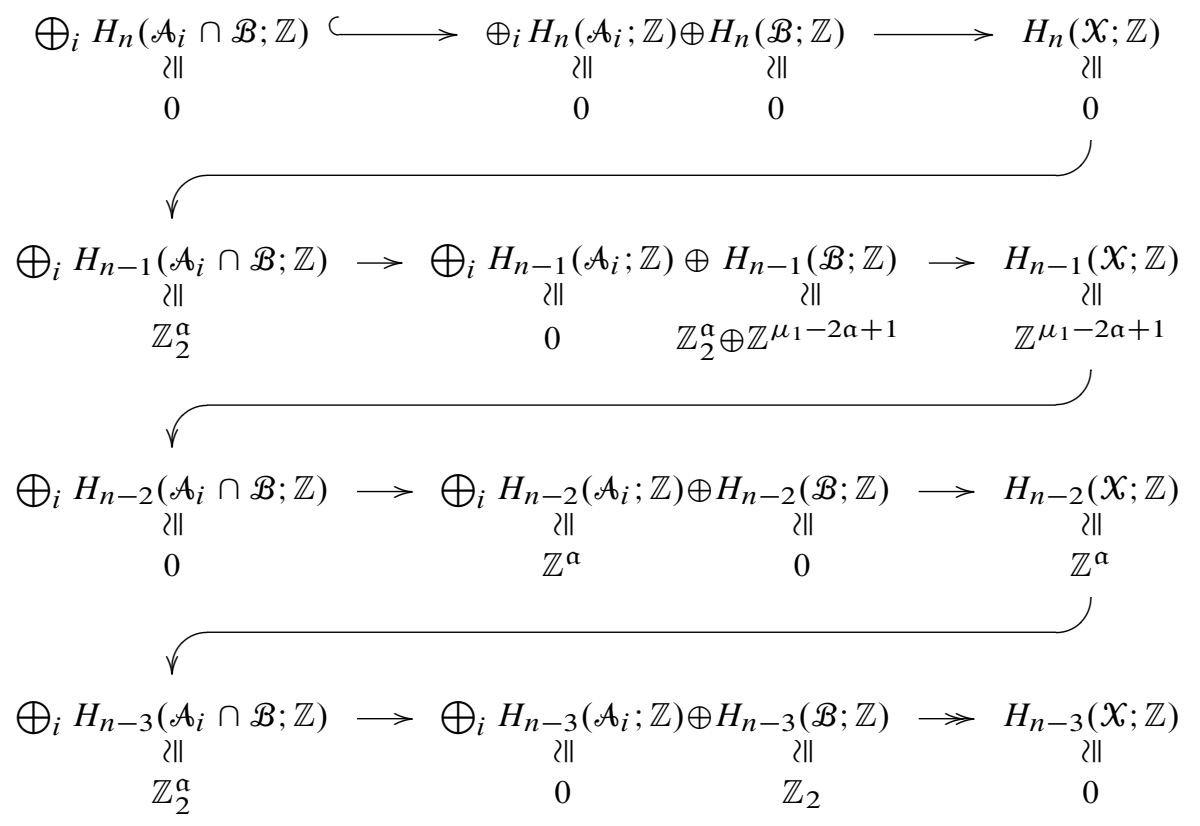

\section{Homology of $\mathcal{M}$}

Now we will compute the homology of $\mathcal{M}$. First we will see that to recover the homotopy type of $\mathcal{M}$ we only need to glue to $\mathcal{X}$ the preimage by $\pi$ of some disks in $\Sigma_{s}$ (the so-called Lefschetz thimbles). Then we will proceed as before, computing the associated Mayer-Vietoris sequence with coefficients in $\mathbb{Z}_{2}$ and then doing a detailed study of the topology in order to lift it to integral coefficients. In this process, we will find some ambiguity: there are two possible values for the homology of $\mathcal{M}$. We prove directly when these two cases will hold. In the final step of this section, we prove that the homology of $\mathcal{M}$ is torsion free. The argument needs the beginning of classification of the singularities treated in this paper, and then a case-by-case study.

9.1. Coefficients in $\mathbb{Z}_{2}$. Recall that $\Sigma_{s}$ is the Milnor fibre of $\Sigma$, and has the homotopy type of a bouquet of $\mu_{0}$ spheres. The functions $g_{1}, \ldots, g_{n-3}, \operatorname{det}(H(f))$ define a 2-dimensional i.c.i.s. $\Sigma[2]_{0}$ with Milnor number $\mu_{1}$ (see Definition 2.5). We may assume that the ball $B_{\epsilon}$ that we are using to compute the Milnor fibre of $f$ is also a Milnor ball for the i.c.i.s.

We need to remind some classical results of i.c.i.s., in particular the definition of Lefschetz thimbles (see Chapter 5 in [11] and [2] for details). There is a disk 
$D_{\delta}$ of centered at the origin of $\mathbb{C}$ such that $\Sigma_{s}$ is homotopy equivalent to $\Sigma_{s} \cap$ $\left(\operatorname{det}\left(H\left(f_{S}\right)\right)\right)^{-1}\left(D_{\delta}\right)$ and that the restriction

$$
\psi:=\left.\operatorname{det}\left(H\left(f_{s}\right)\right)\right|_{\Sigma_{s} \cap\left(\operatorname{det}\left(H\left(f_{s}\right)\right)\right)^{-1}\left(D_{\delta}\right)}: \Sigma_{s} \cap\left(\operatorname{det}\left(H\left(f_{s}\right)\right)\right)^{-1}\left(D_{\delta}\right) \rightarrow D_{\delta}
$$

has precisely isolated critical points with Milnor numbers adding precisely $\mu_{0}+\mu_{1}$. The number a counts the number of critical points in the 0 -fibre of the mapping above. They are all of Morse-type.

Take $\eta$ so small that the disk $D_{\eta}$ only contains 0 as critical value of the restriction to $\psi^{-1}\left(D_{\eta}\right)$. Let $*$ be a point at the boundary of this disc. Draw a system of nonintersecting paths $\left\{\gamma_{i}\right\}_{i}$ joining $*$ with each of the critical values of the mapping $\psi$. Each path corresponds to a vanishing cycle of $\psi$, which is an embedded $\mathbb{S}^{2}$ in $\psi^{-1}(*)$, and that can be continued along the path $\gamma_{i}$ until it collapses to the corresponding critical point of $\psi$. The Lefschetz thimble $C_{i}$ along the path $\gamma_{i}$ is defined to be the union of the continuations of the vanishing cycle along $\gamma_{i}$ until it collapses to a point. It is homeomorphic to a 3-dimensional disk, with boundary the vanishing cycle.

The space $\Sigma_{s}$ is homotopy equivalent to the result of attaching to

$$
\left(\left.\operatorname{det}\left(H\left(f_{s}\right)\right)\right|_{\Sigma_{s}}\right)^{-1}\left(D_{\eta}\right)
$$

the Lefschetz thimbles associated to the critical points of $\left.\operatorname{det}\left(H\left(f_{s}\right)\right)\right|_{\Sigma_{s}}$ not contained in the zero level. There are exactly $\mu_{0}+\mu_{1}-a$ such Lefschetz thimbles (see [11]). Since the Lefschetz thimbles are 3-disks they are attached along 2spheres to the boundary of $\left(\left.\operatorname{det}\left(H\left(f_{s}\right)\right)\right|_{\Sigma_{s}}\right)^{-1}\left(D_{\eta}\right)$, which is 5-dimensional. Hence, a transversality argument ensures that all the attaching spheres are disjoint. Denote by $C_{1}, \ldots, C_{\mu_{0}+\mu_{1}-a}$ the attached Lefschetz thimbles. We have found a homotopy equivalence

$$
M^{\prime}:=\left(\left.\operatorname{det}\left(H\left(f_{s}\right)\right)\right|_{\Sigma_{s}}\right)^{-1}\left(D_{\eta}\right) \cup\left(\bigcup_{i=1}^{\mu_{0}+\mu_{1}-\mathfrak{a}} C_{i}\right) \hookrightarrow \Sigma_{s},
$$

which in fact (since we are working with $C W$-complexes) is a deformation retract.

Since we have a locally trivial fibration

$$
\pi: \mathcal{M} \backslash \pi^{-1}\left(\operatorname{det}\left(H\left(f_{s}\right)\right)^{-1}(0)\right) \rightarrow \Sigma_{s} \backslash \operatorname{det}\left(H\left(f_{s}\right)\right)^{-1}(0)
$$

we can lift the deformation retract (20) to a deformation retract

$$
\mathcal{M}^{\prime}:=\pi^{-1}\left(M^{\prime}\right) \hookrightarrow \mathcal{M}
$$

We will compute the homology of $\mathcal{M}^{\prime}$ using a Mayer-Vietoris sequence. By the previous deformation retract we identify the homology of $\mathcal{M}^{\prime}$ and $\mathcal{M}$. Denote $\pi^{-1}\left(C_{i}\right)$ by $\ell_{i}$. Since $C_{i}$ is contractible the fibration over it is trivial, and, hence, $\ell_{i}$ 
and $\pi^{-1}\left(\partial C_{i}\right)$ are homotopy equivalent to $C_{i} \times \mathbb{S}^{n-4}$, and $\partial C_{i} \times \mathbb{S}^{n-4} \cong \mathbb{S}^{2} \times \mathbb{S}^{n-4}$. Decompose $\mathcal{M}^{\prime}$ as

$$
\mathcal{M}^{\prime}=X \cup\left(\bigcup_{i=1}^{\mu_{0}+\mu_{1}-\mathfrak{a}} e_{i}\right) .
$$

The associated Mayer-Vietoris sequence (with coefficients in $\mathbb{Z}_{2}$ ) is what equation (24) shows, for some $e \in \mathbb{N}$. Recall that only the last column was unknown.

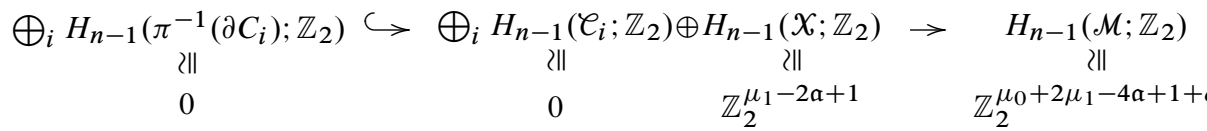

$$
\begin{aligned}
& \sqrt{0}
\end{aligned}
$$

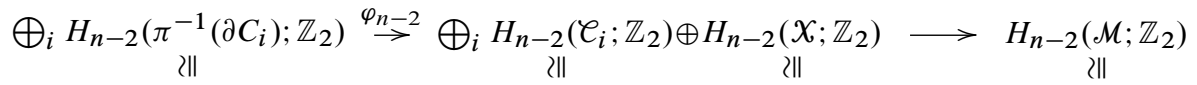

$$
\begin{aligned}
& \begin{array}{llll}
\mathbb{Z}_{2}^{\mu_{0}+\mu_{1}-\mathfrak{a}} & 0 & \mathbb{Z}_{2} \oplus \mathbb{Z}_{2}^{\mathfrak{a}-1} & \mathbb{Z}_{2}^{e}
\end{array} \\
& \begin{array}{cccc}
\bigoplus_{i} H_{n-3}\left(\pi^{-1}\left(\partial C_{i}\right) ; \mathbb{Z}_{2}\right) & \rightarrow \bigoplus_{i} H_{n-3}\left(\mathcal{C}_{i} ; \mathbb{Z}_{2}\right) \oplus H_{n-3}\left(\mathcal{X} ; \mathbb{Z}_{2}\right) & \text { 2\| } & H_{n-3}\left(\mathcal{M} ; \mathbb{Z}_{2}\right) \\
0 & 0 & 0 & 2 \|
\end{array} \\
& \sqrt{ }
\end{aligned}
$$

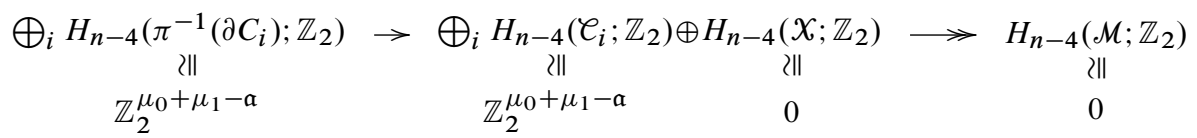

The fact that $\bigoplus_{i} H_{n-4}\left(\pi^{-1}\left(\partial C_{i}\right) ; \mathbb{Z}_{2}\right) \rightarrow \bigoplus_{i} H_{n-4}\left(\mathscr{C}_{i} ; \mathbb{Z}_{2}\right)$ is an isomorphism determines $H_{n-3}\left(\mathcal{M} ; \mathbb{Z}_{2}\right)$ and $H_{n-4}\left(\mathcal{M} ; \mathbb{Z}_{2}\right)$. Given $e, H_{n-4}\left(\mathcal{M} ; \mathbb{Z}_{2}\right)$ is determined by Euler characteristic.

We will find out what are the possible values for $e$. We have given a basis $\left\{Z_{i}\right\}_{i=1}^{a}$ of $H_{n-2}\left(X ; \mathbb{Z}_{2}\right)$ in Subsection 8.1.

\section{Lemma 9.1. The composition}

$$
\tau: \bigoplus_{i} H_{n-2}\left(\pi^{-1}\left(\partial C_{i}\right) ; \mathbb{Z}_{2}\right) \stackrel{\varphi_{n-2}}{\longrightarrow} H_{n-2}\left(X ; \mathbb{Z}_{2}\right) \rightarrow H_{n-2}\left(X ; \mathbb{Z}_{2}\right) /\left(\left[Z_{1}\right]\right)
$$

is surjective. 
Proof. For each one of the a singular points $p_{i}$ of $\operatorname{det}\left(H\left(f_{s}\right)\right)^{-1}(0)$ there is a vanishing cycle $E_{i}$ which is a embedded 2-sphere in $\operatorname{det}\left(H\left(f_{s}\right)\right)^{-1}(u)$. The parameters $s$, $u, \zeta$ (see Section 5) can be chosen so that $\operatorname{det}\left(H\left(f_{s}\right)\right)^{-1}(u) \cap A_{i}(\zeta)$ is a tubular neighbourhood of $E_{i}$ in $\operatorname{det}\left(H\left(f_{s}\right)\right)^{-1}(u)$.

The sphere $\partial C_{k}$ can be chosen to be embedded in $\operatorname{det}\left(H\left(f_{s}\right)\right)^{-1}(u)$ and, after a perturbation, transverse to $E_{i}$ for any $i$. Let

$$
\iota_{k}: \partial C_{k} \hookrightarrow \operatorname{det}\left(H\left(f_{s}\right)\right)^{-1}(u)
$$

denote the embedding. Let $b_{k, i}$ the number of intersection points of $\partial C_{k}$ and $E_{i}$. Choosing the tubular neighbourhoods of $E_{i}$ small enough we find that $\iota_{k}^{-1}\left(A_{i}(\zeta)\right)$ is a disjoint union of disks $D_{k, i, j}$ with $j \in\left\{1, \ldots, b_{k, i}\right\}$, and the boundary of each of them represents the generator of $H_{1}\left(B_{u} ; \mathbb{Z}\right)$. By Remark 6.5 the number $b_{k}:=\sum_{i} b_{k, i}$ is even: otherwise the image in $H_{1}\left(B_{u} ; \mathbb{Z}\right)$ of the boundary

$$
\partial\left(C_{k} \backslash\left(\bigcup_{i, j} D_{k, i, j}\right)\right)
$$

would be a non-zero homology class. We claim the following equality

$$
\tau\left(\left[\pi^{-1}\left(\partial C_{k}\right)\right]\right)=\sum_{i=1}^{a} b_{k, i}\left[Z_{i}\right] .
$$

Let us finish the proof assuming this claim.

Any Lefschetz thimble $C_{k}$ gives rise to a class $\left[\partial C_{k}\right] \in H_{2}\left(\operatorname{det}\left(H\left(f_{s}\right)\right)^{-1}\left(D_{\xi}\right) ; \mathbb{Z}\right)$. It is easy to check that its image by the connecting homomorphism $\delta_{2}$ is equal to

$$
\delta_{2}\left(\left[\partial C_{k}\right]\right)=\sum_{i=1}^{a} b_{k, i}\left[\psi_{i}\right]=\sum_{i=2}^{a} b_{k, i}\left(\left[\psi_{i}\right]-\left[\psi_{1}\right]\right)
$$

where $\psi_{i}$ is a generator of $H_{1}\left(\partial A_{i}(\zeta) \cap B ; \mathbb{Z}\right)$ for any $i$. The first equality is by construction of the connecting homomorphism and the second is true because $\sum_{i} b_{k, i}$ is even and, hence we have the equality $b_{k, 1}=\sum_{i=2}^{a} b_{k, i}$ in $\mathbb{Z}_{2}$.

Let $\alpha_{1}$ be the first mapping of the 1 st row of the sequence (14). Define the isomorphism

$$
\theta: H_{n-2}\left(\mathcal{X}, \mathbb{Z}_{2}\right) /\left(\left[Z_{1}\right]\right) \rightarrow \operatorname{ker}\left(\alpha_{1}\right)
$$

given by $\theta\left(\left[Z_{i}\right]\right):=\left[\psi_{i}\right]-\left[\psi_{1}\right]$. Any element $\left[Z^{\prime}\right] \in H_{n-2}\left(\mathcal{X} ; \mathbb{Z}_{2}\right) /\left(\left[Z_{1}\right]\right)$ corresponds to an element in $\operatorname{ker}\left(\alpha_{1}\right)$, which is the image by $\delta_{2}$ of a class $[Y] \in$ $H_{2}\left(\operatorname{det}\left(H\left(f_{s}\right)\right)^{-1}\left(D_{\xi}\right) ; \mathbb{Z}\right)$. Such a class can be expressed as a sum

$$
[Y]=\sum_{k=1}^{\mu_{0}+\mu_{1}-\mathfrak{a}} m_{k}\left[\partial C_{k}\right]
$$


The coincidence of the coefficients in the last terms of equations (25) and (26) give the equality $\tau([Y])=\left[Z^{\prime}\right]$.

Now we prove the claim. Choose a point $x_{0} \in \partial C_{k} \backslash \bigcup_{i, j} D_{k, i, j}$ and choose a disk $D_{0}$ around it in $\partial C_{k}$ disjoint to the disks $D_{k, i, j}$. Deform the immersion $\left.\iota_{k}\right|_{D_{0}}$ so that the embedding of its boundary remains fixed, it meets $E_{1}$ transversely precisely at $b_{k}$ points, all different from $x_{0}$, and it is disjoint from $E_{j}$ for any $j \neq 1$. After this deformation the intersection $\left.\iota_{k}\right|_{D_{0}} ^{-1}\left(A_{1}(\zeta)\right)$ consists of $b_{k}$ disjoint disks $\left\{D_{k, i, j}^{\prime}\right\}_{i \in\{1, . ., a\}, j \in\left\{1, \ldots, b_{k, i}\right\}}$ (we choose the indexing to make it easy to make a bijection with the disks $D_{k, i, j}$ ).

Choose non-intersecting paths $\alpha_{k, i, j}$ in $D_{0} \backslash\left(\bigcup_{i, j} \dot{D}_{k, i, j}^{\prime}\right)$ joining $x_{0}$ with a point $y_{k, i, j} \in \partial D_{k, i, j}^{\prime}$. Choose non-intersecting paths

$$
\beta_{k, i, j}:[0,1] \rightarrow \partial C_{k} \backslash\left(\bigcup_{k, i, j} \alpha_{k, i, j}([0,1]) \cup \dot{D}_{k, i, j}^{\prime} \cup \dot{D}_{k, i, j}\right)
$$

joining $\partial D_{k, i, j}^{\prime}$ with $\partial D_{k, i, j}$. For a schematic picture, see Figure 3.

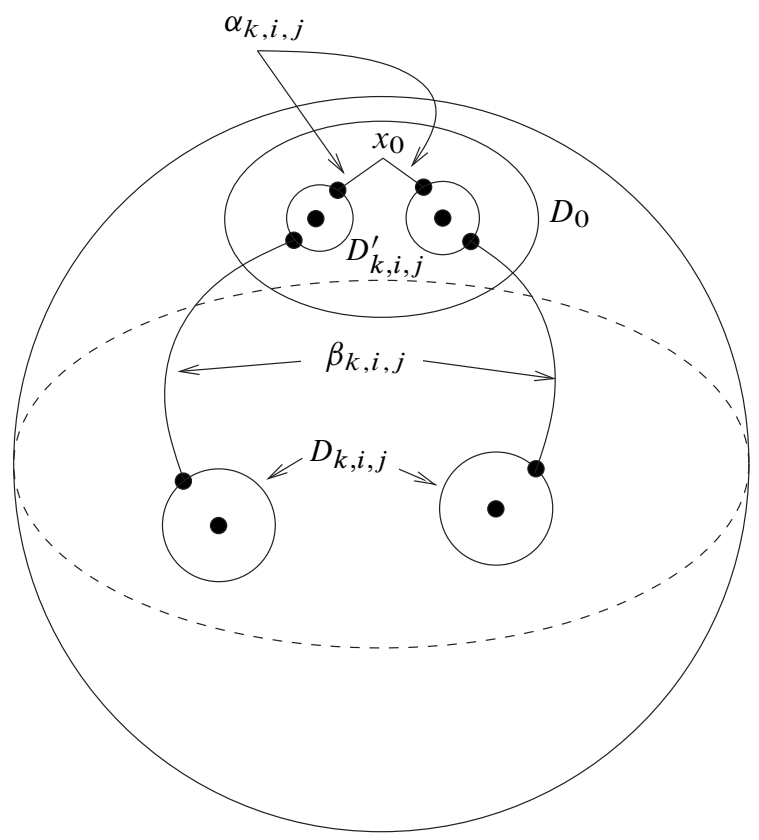

Figure 3. The system of paths in $\partial C_{k}$.

The complement of $\bigcup_{k, i, j}\left(\beta_{k, i, j}([0,1]) \cup \alpha_{k, i, j}([0,1]) \cup D^{\prime}{ }_{k, i, j} \cup D_{k, i, j}\right)$ is a topological disk $G$. Since $G$ is contained in $B_{u}$ we can restrict the fibration (13) to $G$ and obtain a trivial fibration of pairs with fibre homotopic to $\left(\mathbb{S}^{n-3}, \mathbb{S}^{n-4}\right)$, with 
$\mathbb{S}^{n-4}$ embedded as an equator. Consider a mapping

$$
\sigma: G \times \mathbb{S}^{n-3} \rightarrow \mathscr{B}
$$

such that $\sigma\left(\{g\} \times \mathbb{S}^{n-3}\right)$ generates the $(n-3)$-homology of the fibre over $\iota(g)$ by the fibration (13). Denote by $H^{+}$one hemisphere of $\mathbb{S}^{n-3}$. The restriction

$$
\psi: G \times H^{+} \rightarrow \mathscr{B} \subset \mathcal{X}
$$

defines a singular chain in $X$.

Let $Z_{k, i, j}^{\prime}$ be the chain associated to $\beta_{k, i, j}$ by the procedure given in 8.1. Adding and subtracting $\mathbb{S}^{n-2}$-hemispheres $K_{k, i, j}$ and $K_{k, i, j}^{\prime}$ for any $i, j$ (see the procedure in 8.1), the chain $\partial \Upsilon_{k}+\partial \psi$ is shown to be equal to a sum

$$
\sum_{i, j} Z_{i, j}^{\prime}+\sum_{i=1}^{a} Y_{i}
$$

where $Y_{i}$ is a closed chain contained in $\mathcal{A}_{i}$.

The $(n-2)$ nd row of the sequence (18) shows that, for any $i$, any class in $H_{n-2}\left(\mathcal{X} ; \mathbb{Z}_{2}\right)$ supported by a chain contained in $\mathcal{A}_{i}$ is a multiple of $\left[Z_{1}\right]$. On the other hand, by Lemma 8.2 there exists $c_{i, j} \in \mathbb{Z}_{2}$ such that $\left[Z_{i . j}^{\prime}\right]=\left[Z_{i}\right]+c_{i, j}\left[Z_{1}\right]$. This proves the claim.

This means that the only possible values for $e$ are 0 and 1 . We will now characterize the cases in which each value is obtained.

Lemma 9.2. If $\operatorname{corank}\left(H\left[f_{0}\right](0)\right) \geq 3$, then $e=0$.

Proof. Consider the unfolding

$$
F\left(x_{1}, \ldots, x_{n}, b,\left(c_{i, j}\right)\right):=\left(G_{1, b}, \ldots, G_{n-3, b}\right)\left(h_{i, j}+c_{i, j}\right)\left(G_{1, b}, \ldots, G_{n-3, b}\right)^{t}
$$

given in (1). If $\operatorname{corank}\left(H\left[f_{0}\right](0)\right) \geq 3$ there exists a parameter $s_{0} \in S$ and a point $x \in \Sigma_{s_{0}}$ such that the germ $f_{s_{0}}$ at $x$ is right-equivalent to a germ of the form

$$
\left(y_{1}, y_{2}, y_{3}, \ldots\right) \cdot\left(\begin{array}{ccc|c}
l_{1} & l_{2} & l_{3} & \\
l_{2} & l_{4} & l_{5} & 0 \\
l_{3} & l_{5} & l_{6} & \\
\hline & 0 & & \mathrm{Id}
\end{array}\right) \cdot\left(\begin{array}{c}
y_{1} \\
y_{2} \\
y_{3} \\
\vdots
\end{array}\right),
$$

where the $l_{i}$ 's are generic linear forms and the $y_{i}$ 's are variables. The Milnor fibre of such germ function is the suspension of the Milnor fibre $M$ of

$$
\left(y_{1}, y_{2}, y_{3}\right) \cdot\left(\begin{array}{lll}
l_{1} & l_{2} & l_{3} \\
l_{2} & l_{4} & l_{5} \\
l_{3} & l_{5} & l_{6}
\end{array}\right) \cdot\left(\begin{array}{l}
y_{1} \\
y_{2} \\
y_{3}
\end{array}\right),
$$


and this one can be computed by projecting to the variables $\left(y_{1}, y_{2}, y_{3}\right)$. This projection is a fibration over $\mathbb{C}^{3} \backslash\{0\} \approx \mathbb{S}^{5}$ whose fibre is contractible since it is the set of solutions of a system of linear equations. So $M$ has the homotopy type of $\mathbb{S}^{5}$ and $H_{4}(M)=0$. The general case is a suspension of this one. Hence the Milnor fibre of the germ $f_{s_{0}}$ at $x$ is homotopic to $\mathbb{S}^{n-1}$ and its $(n-2)$-homology vanishes.

Since $\Sigma_{s_{0}}$ is smooth at $x$ its versal deformation is trivial. Hence the unfolding given by (1) for the germ $\left(f_{s_{0}}\right)_{x}$ is of the form:

$$
F\left(x_{1}, \ldots, x_{n},\left(c_{i, j}\right)\right):=\left(G_{1, s_{0}}, \ldots, G_{n-3, s_{0}}\right)\left(h_{i, j}+c_{i, j}\right)\left(G_{1, s_{0}}, \ldots, G_{n-3, s_{0}}\right)^{t} .
$$

with $\left(c_{i, j}\right) \in \operatorname{SM}(n-3)$. Observe that this unfolding can be obtained from the unfolding (27) by pullback and localisation near $x$. Let $B\left(x, \epsilon_{0}\right)$ be a Milnor ball for $\left(f_{s_{0}}\right)_{x}$ contained in the Milnor ball $B_{\epsilon}$ of $f$. If $s$ is generic and very close to $s_{0}$ and $t$ is small enough then

$$
\iota: f_{s}^{-1}(t) \cap B\left(x, \epsilon_{0}\right) \hookrightarrow f_{s}^{-1}(t) \cap B_{\epsilon}
$$

is an inclusion of the Milnor fibre of $\left(f_{s_{0}}\right)_{x}$ into the Milnor fibre of $f$.

Since corank $\left(H\left(f_{s_{0}}\right)\right)(x) \geq 2$, if $s$ is close to $s_{0}$ there exists at least a point $p_{i}$ of $\Sigma[2]_{s}$ contained in $B\left(x, \epsilon_{0}\right)$. If $e \neq 0$, that is, if $H_{n-2}\left(\mathcal{M} ; \mathbb{Z}_{2}\right) \neq 0$ then, by Proposition 3.1 with coefficients in $\mathbb{Z}_{2}$ there is an $\mathbb{S}^{n-2}$ in $\mathcal{A}_{i} \subset f_{s}^{-1}(t) \cap$ $B_{\epsilon}$ representing a non-trivial homology class in the Milnor fibre of $f$. But this is impossible: the space $\mathcal{A}_{i}$ is contained in $f_{s}^{-1}(t) \cap B\left(x, \epsilon_{0}\right)$ and the $(n-2)$-homology of this space is trivial. Then there is a chain in $f_{s}^{-1}(t) \cap B\left(x, \epsilon_{0}\right)$ whose boundary is equal to the candidate $\mathbb{S}^{n-2}$. The chain is then contained in $f_{s}^{-1}(t) \cap B_{\epsilon}$, and this shows that the homology class represented by the candidate $\mathbb{S}^{n-2}$ is in fact trivial.

Now we will see that, in the case where corank $\left(H\left[f_{0}\right](0)\right)=2$, the number $e$ turns out to be 1 . Recall that $\mathcal{A}_{i}$ is homotopic to $\mathbb{S}^{n-2}$, denote by $Z$ the generator of $H_{n-2}\left(\mathcal{A}_{i} ; \mathbb{Z}\right)$. Since we have an inclusion $i: \mathcal{A}_{i} \hookrightarrow \mathcal{M}$, we need to check that $i_{*}(Z) \neq 0$.

Lemma 9.3. If $f$ is of the form

$$
f=\left(g_{1}, g_{2}\right) \cdot\left(\begin{array}{ll}
h_{1,1} & h_{1,2} \\
h_{1,2} & h_{2,2}
\end{array}\right) \cdot\left(\begin{array}{l}
g_{1} \\
g_{2}
\end{array}\right),
$$

then $i_{*}(Z) \neq 0$

Proof. Let $\omega$ be a closed differential form defined in $\mathbb{C}^{2} \backslash\{0\}$ such that $\int_{\mathbb{S}^{3}} \omega \neq 0$ for a sphere $\mathbb{S}^{3}$ around the origin in $\mathbb{C}^{2}$. Consider the map

$$
\phi: \mathbb{C}^{n} \rightarrow \mathbb{C}^{2}
$$

defined by $\phi:=\left(g_{1}, g_{2}\right)$. 
Then $\Omega:=\phi^{\star} \omega$ is a closed differential form defined over the Milnor fibre of $f$. The change of variables formula gives the inequality $\int_{i_{\star}(Z)} \phi^{\star} \omega \neq 0$

Now let us generalize this argument for the case where the corank is two, but the dimension is higher:

Lemma 9.4. If $\operatorname{corank}\left(H\left[f_{0}\right](0)\right)=2$, then $e=1$.

Proof. We may assume (see 2.1) that $f$ is of the form

$$
f=\left(g_{1}, g_{2}\right) \cdot\left(\begin{array}{ll}
h_{1,1} & h_{1,2} \\
h_{1,2} & h_{2,2}
\end{array}\right) \cdot\left(\begin{array}{l}
g_{1} \\
g_{2}
\end{array}\right)+g_{3}^{2}+\cdots+g_{n-3}^{2} .
$$

We consider the unfolding $F$ defined in (6). Clearly there are parameters $s$ such that the functions $\left(h_{1,1, s}, h_{1,2, s}, h_{2,2, s}, G_{1, s}, \ldots, G_{n-3, s}\right)$ vanish at the origin and form a holomorphic coordinate system around it. In this case the local Milnor fibre of the deformed function $F_{S}$ at the origin has the homotopy type of a $(n-2)$-sphere.

Let $\epsilon$ and $\delta$ be radii for the Milnor fibration of $f$. Let $\epsilon^{\prime}$ and $\delta^{\prime}$ be radii for the Milnor fibration of $f_{s}$ at the origin. By Theorem 2.2 we have that $F_{s}^{-1}\left(\delta^{\prime}\right) \cap B_{\epsilon}$ is diffeomorphic to the Milnor fibre of $f$. Let $Z$ be a cycle in the local Milnor fibre $F_{s}^{-1}\left(\delta^{\prime}\right) \cap B_{\epsilon^{\prime}}$ generating the $(n-2)$-homology group. In order to show that $e=1$ it is enough to show that the homology class $[Z]$ is nonzero considered in the bigger space $F_{s}^{-1}\left(\delta^{\prime}\right) \cap B_{\epsilon}$. For this it suffices to find a closed $(n-2)$-differential form $\Omega$, defined in $F_{s}^{-1}\left(\delta^{\prime}\right) \cap B_{\epsilon}$ such that $\int_{Z} \Omega \neq 0$.

In order to define such a form, choose a positive function $\beta: \mathbb{C} \mapsto \mathbb{R} \subseteq \mathbb{C}$ such that $\left.\beta\right|_{D(0, \eta / 2)} \equiv 0$ and $\left.\beta\right|_{\mathbb{C} \backslash D(0, \eta)} \equiv 1$ for a sufficiently small radius $\eta$. Now take $\omega$ a closed 3-form in $\mathbb{C}^{2} \backslash\{0\}$ that generates the de Rham cohomology in degree 3 .

We have the function

$$
\psi: \mathbb{C}^{n} \backslash V\left(G_{1, s}, G_{2, s}\right) \rightarrow \mathbb{C}^{2} \backslash\{0\}, \quad x \mapsto\left(G_{1, s}(x), G_{2, s}(x)\right) .
$$

Define

$$
\Omega:=\psi^{*} \omega \wedge \beta\left(G_{3, s}^{2}+\cdots+G_{n-3, t}^{2}-\delta^{\prime}\right) d G_{3, s} \wedge \cdots \wedge d G_{n-3, s} .
$$

Let us check that $\Omega$ is defined in all $F_{s}^{-1}\left(\delta^{\prime}\right) \cap B_{\epsilon}$ : the form $\psi^{\star} \omega$ is only defined in $\mathbb{C}^{n} \backslash V\left(G_{1, s}, G_{2, s}\right)$, but the factor $\beta\left(G_{3, s}^{2}-\cdots-G_{n-3, s}^{2}-\delta^{\prime}\right)$ is identically zero when $G_{3, s}^{2}-\cdots-G_{n-3, s}^{2}-\delta^{\prime}$ is small enough.

In order to check that $\Omega$ is closed notice that since $\omega$ is closed, so is $\psi^{*} \omega$. Hence it is sufficient to show that

$$
\beta\left(G_{3, s}^{2}+\cdots+G_{n-3, t}^{2}-\delta^{\prime}\right) d G_{3, s} \wedge \cdots \wedge d G_{n-3, s}
$$


is closed. A chain rule argument shows the equality

$$
d \beta\left(G_{3, s}^{2}+\cdots+G_{n-3, t}^{2}-\delta^{\prime}\right)=\frac{\partial \beta}{\partial z}\left(G_{3, s}^{2}-\cdots-G_{n-3, s}^{2}\right) \sum_{i=3}^{n-3} 2 G_{i, s} d G_{i, s},
$$

which means that

$$
\begin{gathered}
d \Omega=\psi^{\star} w \wedge \frac{\partial \beta}{\partial z}\left(G_{3, s}^{2}+\cdots+G_{n-3, t}^{2}-\delta^{\prime}\right)\left(\sum_{i=3}^{n-3} 2 G_{i, s} d G_{i, s}\right) \\
\wedge d G_{3, s} \wedge \cdots \wedge d G_{n-3, s}=0 .
\end{gathered}
$$

Finally we will check that the form $\Omega$ integrated against the cycle $Z$ gives a nonzero result. We start by giving an explicit description of $Z$. Let $Z^{\prime}$ be the cycle that generates the 3-homology of

$$
\left\{\left(G_{1, s}, G_{2, s}\right)\left(h_{i, j, s}\right)\left(G_{1, s}, G_{2, s}\right)^{t}=\delta^{\prime}\right\} \cap B_{\epsilon^{\prime}} .
$$

Define the family of mappings

$$
\alpha[\delta]: \mathbb{C}^{5} \rightarrow \mathbb{C}^{5}, \quad\left(x_{1}, x_{2}, x_{3}, x_{4}, x_{5}\right) \mapsto \sqrt[3]{\frac{8}{3}}\left(x_{1}, x_{2}, x_{3}, x_{4}, x_{5}\right)
$$

which depend on a parameter $\delta$. The cycle $Z$ admits the following parametrisation: since the functions

$$
\left(h_{1,1, s}, h_{1,2, s}, h_{2,2, s}, G_{1, s}, \ldots, G_{n-3, s}\right)
$$

form a holomorphic coordinate system at the origin the vanishing of the first 5 of them defines a germ $(M, O)$ of $(n-5)$-dimensional complex manifold at the origin. Let $B\left(0, \sqrt{\delta^{\prime}}\right)$ denote the ball of radius $\sqrt{\delta^{\prime}}$ centered at the origin of $\mathbb{R}^{n-5}$, being $\mathbb{R}^{n-5}$ the real locus of $(M, O)$. The parametrisation is given by

$$
\begin{aligned}
Z^{\prime} \times B\left(0, \sqrt{\delta^{\prime}}\right) & \rightarrow Z, \\
\left(p, y_{3}, \ldots y_{n-3}\right) & \mapsto\left(\alpha\left[\delta^{\prime}-y_{3}^{2}-\cdots-y_{n-3}^{2}\right](p), y_{3}, \ldots y_{n-3}\right) .
\end{aligned}
$$

We compute $\int_{Z} \Omega$ in what follows. The first equality is due to the change of variables formula, the second follows from Fubini's theorem, and the third is because of Lemma 9.3.

$$
\begin{aligned}
\int_{Z} \Omega= & \int_{Z^{\prime} \times B\left(0, \sqrt{\delta^{\prime}}\right)} \psi^{*} \omega \wedge \beta\left(G_{3, s}^{2}+\cdots+G_{n-3, s}^{2}-\delta^{\prime}\right) d G_{3, s} \wedge \cdots \wedge G_{n-3, s} \\
= & \int_{B\left(0, \sqrt{\delta^{\prime}}\right)} \beta\left(G_{3, s}^{2}-\cdots+G_{n-3, s}^{2}-\delta^{\prime}\right) \\
& \cdot \int_{Z^{\prime}} \alpha\left[G_{3, s}^{2}+\cdots+G_{n-3, s}^{2}-\delta^{\prime}\right]^{*} \psi^{*} \omega d G_{3, s} \wedge \cdots \wedge d G_{n-3, s} \\
= & \int_{B\left(0, \sqrt{\delta^{\prime}}\right)} \beta\left(G_{3, s}^{2}+\cdots+G_{n-3, \delta^{\prime}}^{2}-\delta^{\prime}\right) d G_{3, s} \wedge \cdots \wedge d G_{n-3, s}
\end{aligned}
$$

which is nonzero. 
Now we can easily prove Lemma 8.1 using an example:

Proof of Lemma 8.1. Since we are with coefficients in $\mathbb{Z}_{2}$, if the mapping is not an isomorphism then it is identically zero.

The function $f: \mathbb{C}^{5} \rightarrow \mathbb{C}$ given by

$$
f=\left(x_{1}, x_{2}\right) \cdot\left(\begin{array}{cc}
x_{3} & x_{4} \\
x_{4} & x_{3}-x_{5}^{2}
\end{array}\right) \cdot\left(\begin{array}{l}
x_{1} \\
x_{2}
\end{array}\right)
$$

has finite extended codimension with respect to $\left(x_{1}, x_{2}\right)$ : the critical set is precisely $V\left(x_{1}, x_{2}\right)$ and away from the origin it has singularities of type $D(3, p)$, which are of extended codimension equal to 0 .

By a procedure similar to the one we have used to compute the homotopy type of the Milnor fibre of the $D(3,2)$ singularity, in [5] we have proven that the Milnor fibre of $f$ at the origin is homotopy equivalent to $\mathbb{S}^{3}$.

An easy computation shows that if we take a generic parameter $s$ of the unfolding $F$ associated to $f$ in Section 2 then $F_{s}$ has no Morse points outside $\Sigma_{s}=\Sigma_{0}$, there are precisely 2 points of type $D(3,2)$, and the Milnor number of the i.c.i.s. $\Sigma_{0} \cap\{\operatorname{det}(H(f))=0\}$ is equal to 3 . Let us assume that the mapping in the statement of Lemma 8.1 is identically zero. In this case the previous long exact sequences can be used to compute the homology of the Milnor fibre of $f$, and they give that the 4-homology group is non-zero. This gives a contradiction.

9.2. Integral coefficients. Now we compute the integral homology of $\mathcal{M}$. After the previous computation of the homology with coefficients in $\mathbb{Z}_{2}$ this amounts essentially to compute the torsion. We will prove that the homology is torsionfree. The arguments are somewhat intrincate and we need to consider the cases of $\operatorname{corank}(H(f)(O)) \geq 3$ and of $\operatorname{corank}(H(f)(O))=2$ in a separate way.

From the integer homology of $\mathcal{X}$, it is easy to see by the Mayer-Vietoris sequence that $H_{k}(\mathcal{M} ; \mathbb{Z})=H_{k}(\mathcal{X} ; \mathbb{Z})$ for $k \neq n-1, n-2$.

On the other hand, the group $H_{n-1}(\mathcal{M} ; \mathbb{Z})$ is torsion free since $\mathcal{M}$ is a $(n-1)$ dimensional Stein space. By the Universal Coefficients Theorem and our computation of homology with coefficients in $\mathbb{Z}_{2}$, it is easily obtained that $H_{n-2}(\mathcal{M} ; \mathbb{Z})$ has no 2-torsion: as we have seen in the proof of Lemma 9.4, when $e=1$, the $\mathbb{Z}_{2}$ component of $H_{n-2}\left(\mathcal{M} ; \mathbb{Z}_{2}\right)$ is represented by a torsion free class (its integral against a closed form is non-zero), and hence it comes from a $\mathbb{Z}$ component in $H_{n-2}(\mathcal{M} ; \mathbb{Z})$.

Summarising, the Mayer-Vietoris sequence with coefficients in $\mathbb{Z}$ is as shown in equation (30), where $T$ is a torsion group without 2-torsion. We prove now that $T=0$. We have to deal separately with the cases corank $(H(f)) \geq 3$ and corank $(H(f))=2$.

Proposition 9.5. If $\operatorname{corank}(H(f)(O)) \geq 3$, then $T=0$. 


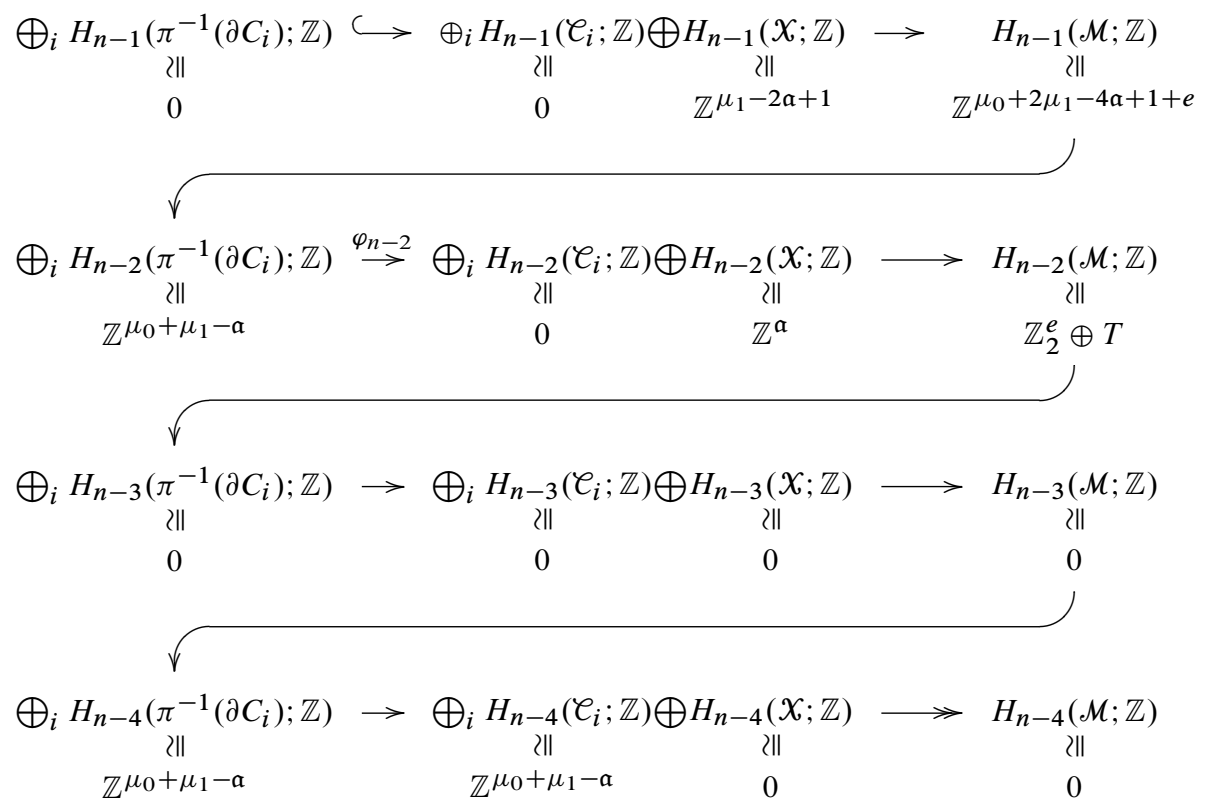

Proof. Let $F: \mathbb{C}^{n} \times S \rightarrow \mathbb{C}$ be the unfolding associated with $f$ in Section 2. By Theorem 2.2 there is a monodromy representation

$$
\rho: \pi_{1}(S \backslash \Delta) \rightarrow \operatorname{Aut}\left(H_{n-2}\left(F_{S}^{-1}(\delta) \cap B_{\epsilon} ; \mathbb{Z}\right)\right) .
$$

By Lemma 2.4, if one of the generators of the form $S_{i}$ of $H_{n-2}(X ; \mathbb{Z})$ maps to zero in $H_{n-2}\left(F_{s}^{-1}(\delta) \cap B_{\epsilon} ; \mathbb{Z}\right)$, then every other generator of the form $S_{j}$ maps to zero too. In the proof Lemma 9.2 we have seen that this is the case. By homology splitting we conclude that any $S_{i}$ is zero in $H_{n-2}(\mathcal{M} ; \mathbb{Z})$.

Now let $z \in H_{n-2}(\mathcal{M} ; \mathbb{Z})$ be a $p$-torsion element with $p \neq 2$. Then $p z=0$, which means that, considered with coefficients in $\mathbb{Z}_{2}$ its class $[z] \in H_{n-2}\left(\mathcal{M} ; \mathbb{Z}_{2}\right)$ must be also zero. This implies that $z$ is homologous to $z^{\prime}=\sum_{i} 2 a_{i} Z_{i}$.

Consider the exact sequence

$0 \rightarrow \bigoplus_{i} H_{n-2}\left(\mathcal{A}_{i} ; \mathbb{Z}\right) \rightarrow H_{n-2}(\mathcal{X} ; \mathbb{Z}) \rightarrow \bigoplus_{i} H_{n-3}\left(\partial \mathcal{A}_{i} ; \mathbb{Z}\right) \rightarrow H_{n-3}(\mathscr{B} ; \mathbb{Z}) \rightarrow 0$

The images of the generators of $H_{n-2}\left(\mathcal{A}_{i} ; \mathbb{Z}\right)$ in $H_{n-2}(\mathcal{X} ; \mathbb{Z})$ have been denoted before by $S_{i}$, and the $Z_{i}$ 's correspond to the generators of the kernel of

$$
\bigoplus_{i} H_{n-3}\left(\partial \mathcal{A}_{i} ; \mathbb{Z}\right) \rightarrow H_{n-3}(\mathcal{B} ; \mathbb{Z})
$$


which is isomorphic to $\mathbb{Z}_{2}^{\mathfrak{a}-1}$. The exactness of the sequence implies that $2 Z_{i}$ can be expressed as a linear combination of the $S_{i}$ 's, but as we have seen before, all of them are zero in $H_{n-2}(\mathcal{M} ; \mathbb{Z})$.

Proposition 9.6. If $\operatorname{corank}(H(f)(O))=2$, then $T=0$.

Proof. Let $z \in H_{n-2}(\mathcal{M} ; \mathbb{Z})$ be a $p$-torsion element with $p \neq 2$. Then we have the following equality with coefficients in $\mathbb{Z}_{2}$ :

$$
0=[z] \in H_{n-2}\left(\mathcal{M} ; \mathbb{Z}_{2}\right) .
$$

As before, this means that homologically, $z$ can be expressed as $z=\sum_{i} a_{i} S_{i}$. Assume that all $S_{i}$ are equal in $H_{n-2}(\mathcal{M} ; \mathbb{Z})$. We would have that, integrating against the form $\Omega$ of Lemma 9.3 and Lemma 9.4 (normalizing it if necessary) we get

$$
\int_{z} \Omega=\sum_{i} a_{i} \int_{S_{i}} \Omega=\sum_{i} a_{i}
$$

which, by the hypothesis of $z$ being of $p$-torsion, means that $\sum_{i} a_{i}=0$, and, hence, that $[z]=0$.

We only need to prove that $S_{i}$ and $S_{j}$ represent the same class in $H_{n-2}(\mathcal{M} ; \mathbb{Z})$ for any $i, j$.

If the functions

$$
\left\{h_{1,1}, h_{1,2}, h_{2,2}, g_{1}, \ldots, g_{n-3}\right\}
$$

form an i.c.i.s at the origin of Milnor number 0 (that is they are smooth and transverse) then there is only one sphere $S_{1}$ and the result is proved. Let us assume that they form an i.c.i.s at the origin of Milnor number at least 1 .

Given a point $s_{0} \in S \backslash \Lambda$ there is a $1-1$ correspondence between points $p_{i}$ of $\Sigma[2]_{s_{0}}$ and spheres $S_{i}$ as above. To a vanishing cycle $\left\{p_{i}, p_{j}\right\}$ (recall Definition 2.6) corresponds a pair of spheres $\left\{S_{i}, S_{j}\right\}$. By Lemmas 2.7 and 2.8 in order to prove that $S_{i}$ and $S_{j}$ represent the same class in $H_{n-2}(\mathcal{M} ; \mathbb{Z})$ for any $i, j$ it is enough to show that there exists a vanishing cycle $\left\{p_{i}, p_{j}\right\}$ such that $S_{i}$ and $S_{j}$ represent the same class in $H_{n-2}(\mathcal{M} ; \mathbb{Z})$. This reduces the proof to the case in which the Milnor number of the i.c.i.s. defined by (31) at the origin is 1 .

The fact that the functions (31) have Milnor number 1 at the origin implies that at least $n-1$ of them must be linearly independent variables (after a suitable change of coordinates). Depending on which one of the functions is not a variable, there are four possible cases, that will be analysed below. In this analysis we will use repeatedly the following fundamental Lemma:

Lemma 9.7. The homology of the Milnor fibre of a germ $f$ only depends on the number of Morse points appearing in a generic value of $s$ of the base space of the versal deformation $S$ and on the topology of the triple $\left(\Sigma_{s}, \Sigma[1]_{s}, \Sigma[2]_{s}\right)$. The 
homology of $\mathcal{M}$ only depends on the topology of the triple. The homology of the Milnor fibre has torsion if and only if the homology of $\mathcal{M}$ has torsion.

Proof. The first and third statements of the Lemma are true by homology splitting.

For the second recall that in Section 5 the spaces $\mathcal{M}$ and $\Sigma_{s}$ are decomposed in three pieces. Each piece of $\mathcal{M}$ is a fibration of spheres over a piece of $\Sigma_{s}$. The topology of the pieces of $\Sigma_{s}$, the dimensions of the fibre spheres and the Gysin sequence of the fibrations only depend on the topology of the triple $\left(\Sigma_{s}, \Sigma[1]_{s}, \Sigma[2]_{s}\right)$. Thus the homology of each of the pieces of $\mathcal{M}$ is determined. The Mayer-Vietoris exact sequences needed to recover the homology of $\mathcal{M}$ in terms of the homology of the pieces are also determined by the topology of the triple.

We continue with the proof of the proposition. The list of cases is the following:

Case 1. Suppose $f=\left(g_{1}, g_{2}\right) \cdot\left(\begin{array}{ll}g_{3} & g_{4} \\ g_{4} & g_{5}\end{array}\right) \cdot\left(\begin{array}{l}g_{1} \\ g_{2}\end{array}\right)$ with $g_{1}, g_{2}, g_{3}, g_{4}$ independent variables (note that the role of $g_{3}$ and $g_{5}$ can be interchanged, and hence this case also studies the case where $g_{1}, g_{2}, g_{4}, g_{5}$ are independent variables). In this case, we can take coordinates such that $g_{i}=x_{i}$ for $i=1, \ldots, 4$, and $g_{5}=a x_{3}+b x_{5}^{2}+\phi$, being $\phi$ a sum of higher order terms.

Consider the following family of functions:

$$
f_{t}=\left(x_{1}, x_{2}\right) \cdot\left(\begin{array}{cc}
x_{3} & x_{4} \\
x_{4} & a x_{3}+b x_{5}^{2}+t \phi
\end{array}\right) \cdot\left(\begin{array}{l}
x_{1} \\
x_{2}
\end{array}\right) .
$$

It is clear that $f_{1}=f$. For any $t$ the singular set $\Sigma$ is smooth, the set $\Sigma[1]$ is the surface given by the suspension of two smooth branches with intersection multiplicity equal to 2, and the set $\Sigma[2]$ is just the origin. After a perturbation the triple $(\Sigma, \Sigma[1], \Sigma[2])$ becomes a triple which has the topology of

$$
\left(\mathbb{C}^{3}, V\left(z_{1}\left(z_{1}+z_{2}^{2}-1\right)+z_{3}^{2}, V\left(z_{1}, z_{2}^{2}-1, z_{3}\right)\right)\right.
$$

independently of $t$. Moreover in the generic perturbation there are no $A_{1}$ points appearing outside $\Sigma$ for any $t$. Therefore, by Lemma 9.7 in order to compute the homology of the Milnor fibre we may assume $t=0$.

Write $f_{0}=x_{1}^{2} x_{3}+2 x_{1} x_{2} x_{4}+a x_{3} x_{2}^{2}+b x_{2}^{2} x_{5}^{2}=\left(x_{1}^{2}+a x_{2}^{2}\right) x_{3}+\left(2 x_{1} x_{2}\right) x_{4}+$ $b x_{2}^{2} x_{5}^{2}$. Since it is quasi-homogenous, we can take infinite Milnor radius and we are reduced to compute the homology of:

$$
\left(x_{1}^{2}+a x_{2}^{2}\right) x_{3}+\left(2 x_{1} x_{2}\right) x_{4}+b x_{2}^{2} x_{5}^{2}=1 .
$$

Projecting to $\left(x_{1}, x_{2}\right)$, we see that there exists a preimage if and only if $\left(x_{1}^{2}+\right.$ $\left.a x_{2}^{2}, x_{1} x_{2}, b x_{2}^{2}\right) \neq(0,0,0)$, that is, everywhere except in the point $\left(x_{1}, x_{2}\right)=(0,0)$. It can be easily checked that the fibre over each point is contractible, and hence the Milnor fibre $F_{f_{0}}$ has the homotopy type of $\mathbb{C}^{2} \backslash\{0\} \approx \mathbb{S}^{3}$. 
Case 2. Suppose

$$
f=\left(g_{1}, g_{2}\right) \cdot\left(\begin{array}{ll}
g_{3} & g_{4} \\
g_{4} & g_{5}
\end{array}\right) \cdot\left(\begin{array}{l}
g_{1} \\
g_{2}
\end{array}\right)
$$

with $g_{1}, g_{2}, g_{3}, g_{5}$ independent variables. We can write

$$
f=\left(x_{1}, x_{2}\right) \cdot\left(\begin{array}{ll}
x_{3} & g_{4} \\
g_{4} & x_{5}
\end{array}\right) \cdot\left(\begin{array}{l}
x_{1} \\
x_{2}
\end{array}\right),
$$

where $g_{4}=a x_{1}+b x_{2}+x_{4}^{2}+\phi$, being $\phi$ again a sum of higher order terms. After an appropriate change of basis in $x_{1}$ and $x_{2}$ we get

$$
\begin{aligned}
f=\left(x_{1}, x_{2}\right) \cdot\left(\begin{array}{cc}
x_{3} & a x_{1}+b x_{2}+x_{4}^{2}+\phi \\
a x_{1}+b x_{2}+x_{4}^{2}+\phi & x_{5}
\end{array}\right) \cdot\left(\begin{array}{l}
x_{1} \\
x_{2}
\end{array}\right) \\
=\left(x_{1}-x_{2}, x_{2}\right) \cdot\left(\begin{array}{cc}
x_{3} & a x_{1}+b x_{2}+x_{3}+x_{4}^{2}+\phi \\
a x_{1}+b x_{2}+x_{3}+x_{4}^{2}+\phi & 2 a x_{1}+2 b x_{2}+x_{3}+x_{5}
\end{array}\right) \\
\cdot\left(\begin{array}{c}
x_{1}-x_{2} \\
x_{2}
\end{array}\right) \\
=\left(x_{2}, x_{1}-x_{2}\right) \cdot\left(\begin{array}{c}
2 a x_{1}+2 b x_{2}+x_{3}+x_{5} \\
a x_{1}+b x_{2}+x_{3}+x_{4}^{2}+\phi
\end{array}\right) \\
\cdot\left(\begin{array}{c}
x_{2} \\
x_{1}-x_{2}
\end{array}\right)
\end{aligned}
$$

which falls into the previous case.

Case 3. Suppose $f=\left(g_{1}, g_{2}\right) \cdot\left(\begin{array}{l}g_{3} \\ g_{4} \\ g_{4}\end{array}\right) \cdot\left(\begin{array}{l}g_{1} \\ g_{2}\end{array}\right)$ with $g_{1}$ and $g_{2}$ are not linearly independent variables. After a change of base, we may assume that $f$ is of the form

$$
f=\left(x_{1}, q\right) \cdot\left(\begin{array}{ll}
x_{3} & x_{4} \\
x_{4} & x_{5}
\end{array}\right) \cdot\left(\begin{array}{c}
x_{1} \\
q
\end{array}\right)
$$

where $q$ has a Taylor development starting by a generic quadric. Like in Case 1, using Lemma 9.7 and an appropriate family $f_{t}$, we may reduce the to the case in which $q=x_{1}^{2}+x_{2}^{2}+x_{3}^{2}+x_{4}^{2}+x_{5}^{2}$.

The triple $(\Sigma, \Sigma[1], \Sigma[2])$ and its deformations $\left(\Sigma_{s}, \Sigma[1]_{s}, \Sigma[2]_{s}\right)$ when we move $s$ in the base $S$ of the unfolding are always contained in the hyperplane $x_{1}=0$. We restrict to this hyperplane and forget the variable $x_{1}$ for the rest of the analysis of this case.

In this hyperplane, the i.c.i.s. $\Sigma$ is given the hypersurface $q=0$, and the singular locus of det $=x_{3} \cdot x_{5}-x_{4}^{2}$ is the $x_{2}$-axis. When we consider the Milnor fibre $q^{-1}(s)$, it intersects the $x_{2}$ axis in two points. These two points correspond to two vanishing cycles $S_{1}, S_{2}$ in the Milnor fibre $\mathscr{F}$ of $\Sigma[1]=V\left(x_{1}, q\right.$, det $)$. Each vanishing cycle $S_{i}$ corresponds to a point $p_{i} \in \Sigma[2]_{s}$, which gives a class $S_{i}$ in $H_{3}(\mathcal{M} ; \mathbb{Z})$. We 
need to prove that the classes $\delta_{1}$ and $\delta_{2}$ are equal. Running in this particular case the geometric considerations made for the proof of Equality (25) in the proof of Lemma 9.1, we observe that if we find a vanishing cycle $S_{3}$ in $\mathcal{F}$ meeting each $S_{1}$ and $S_{2}$ transversely at a point, we can use it and the fibrations above it, in order to express the chain $\delta_{1}-\delta_{2}$ as a boundary.

The critical locus of the germ $(q$, det $): \mathbb{C}^{4} \rightarrow \mathbb{C}^{2}$ consists of four linear components, whose parametrisations are given by $(t, 0,0,0),(0, t, 0, t),(0, t, 0,-t)$ and $(0,0, t, 0)$ respectively. The corresponding components of the discriminant are parametrised as follows: $(t, 0),\left(2 t^{2}, t^{2}\right),\left(2 t^{2},-t^{2}\right)$ and $\left(t^{2},-t^{2}\right)$. Since we are working on the Milnor fibre of $q$, we are looking at the preimage of the set $\left\{(x, y) \in \mathbb{C}^{2} \mid\right.$ $x=1\}$. In that line, the point $(1,0)$ correspond to the values where we want to look for the vanishing cycle touching the two critical points, which are $(1,0,0,0)$ and $(-1,0,0,0)$. In order to track how this cycle vanishes, we will consider the interval $(1, \epsilon)$, where $\epsilon$ ranges from 0 to $\frac{1}{2}$. We will consider the expansion of $q$ and det based at the point $\left(0, \frac{1}{\sqrt{2}}, 0, \frac{1}{\sqrt{2}}\right)$ :

$$
\begin{gathered}
q=x_{2}^{2}+\sqrt{2} x_{3}+x_{3}^{2}+x_{4}^{2}+\sqrt{2} x_{5}+x_{5}^{2}+1, \\
\operatorname{det}=\frac{1}{2}+\frac{1}{\sqrt{2}}\left(x_{3}+x_{5}\right)+x_{3} x_{5}-x_{4}^{2} .
\end{gathered}
$$

For a fixed $\epsilon \in\left[0, \frac{1}{2}\right]$, the fibre over the point $(1, \epsilon)$ is given by

$$
\begin{gathered}
\frac{1}{2} w^{2}+\sqrt{2} w+x_{2}^{2}+\frac{1}{2} z^{2}+x_{4}^{2}=0, \\
x_{2}^{2}+3 x_{4}^{2}+z^{2}=1-2 \epsilon
\end{gathered}
$$

where $w=\left(x_{3}+x_{5}\right), z=x_{3}-x_{5}$.

The real solutions of $x_{2}^{2}+3 x_{4}^{2}+z^{2}=1-2 \epsilon$ are a single point if $\epsilon=\frac{1}{2}$ and a 2-sphere if $\epsilon \in\left[0, \frac{1}{2}\right)$. Fixed $x_{2}, x_{4}$ and $z$, there are two possible choices for $w$, except when the discriminant of $\frac{1}{2} w^{2}+\sqrt{2} w+x_{2}^{2}+\frac{1}{2} z^{2}+x_{4}^{2}$ vanishes, that is, when $x_{2}^{2}+\frac{1}{2} z^{2}+x_{4}^{2}=1$. But this condition, together with $x_{2}^{2}+3 x_{4}^{2}+z^{2}=1-2 \epsilon$ implies $4 x_{4}^{2}+z^{2}=-4 \epsilon$, which does not have real solutions if $\epsilon>0$. Since $\mathbb{S}^{2}$ is simply connected, the only possible double cover over it is two copies of $\mathbb{S}^{2}$. That is, we have two copies of $\mathbb{S}^{2}$ over each point between $(1,0)$ and $\left(1, \frac{1}{2}\right)$; this two spheres collapse when we go to $\left(1, \frac{1}{2}\right)$, and they intersect in two different points at $(1,0)$. This two points of intersection are precisely $(1,0,0,0)$ and $(-1,0,0,0)$, which are the singular points of det at $q=1$. Any of this two spheres is a vanishing cycle as we are looking for.

Case 4. If $f$ is of the form

$$
f=\left(x_{1}, x_{2}, g_{6}\right) \cdot\left(\begin{array}{ccc}
x_{3} & x_{4} & 0 \\
x_{4} & x_{5} & 0 \\
0 & 0 & 1
\end{array}\right) \cdot\left(\begin{array}{l}
x_{1} \\
x_{2} \\
g_{6}
\end{array}\right)
$$


with the linear part of $g_{6}$ linearly dependent with $x_{1}, x_{2}, x_{3}, x_{4}, x_{5}$, the configurations $(\Sigma, \Sigma[1], \Sigma[2])$ and its deformations $\left(\Sigma_{s}, \Sigma[1]_{s}, \Sigma[2]_{s}\right)$ are easily checked to be suspensions of those in the previous case. Since all the method depends on this configuration, this case can be treated in the same way as the previous one.

9.3. The case of $\operatorname{corank}(H(f)(O))=1$. Let us compute the homology of $\mathcal{M}$ in the special case in which corank $(H(f)(O))=1$. If one recalls the decomposition of $\mathcal{M}$ given in Section 5 we observe the equalities $B_{0}=\operatorname{det}\left(H\left(f_{s}\right)\right)^{-1}(0)$ and $\mathcal{X}=\mathscr{B}$. Moreover $B_{0}$ is smooth and diffeomorphic to the Milnor fibre of the i.c.i.s. $\operatorname{det}\left(H\left(f_{0}\right)\right)^{-1}(0)$, and hence we have the homotopy equivalence

$$
B_{0} \approx \bigvee_{\mu_{1}} \mathbb{S}^{2}
$$

The space $\mathscr{B}$ fibres over $B_{0}$ with fibre homotopic to $\mathbb{S}^{n-3}$, and $\mathscr{B}_{u}$ fibres over $B_{0}$ with fibre $\mathbb{S}^{n-4}$. Since $B_{0}$ is simply connected, both fibrations are orientable. Using the Gysin sequence of these fibrations we get that $H_{k}(\mathcal{B} ; \mathbb{Z}) \cong \mathbb{Z}$ for $k=n-3,0$, $H_{k}(\mathscr{B} ; \mathbb{Z}) \cong \mathbb{Z}^{\mu_{1}}$ for $k=n-1,2$, and 0 otherwise. Adding the Lefschetz thimbles as in Subsection 9.2, we obtain that

$$
\begin{gathered}
H_{n-1}(\mathcal{M} ; \mathbb{Z}) \cong \mathbb{Z}^{2 \mu_{1}+\mu_{0}}, \\
H_{n-3}(\mathcal{M} ; \mathbb{Z}) \cong \mathbb{Z}, \\
H_{2}(\mathcal{M} ; \mathbb{Z}) \cong \mathbb{Z}^{\mu_{0}}, \\
H_{0}(\mathcal{M} ; \mathbb{Z}) \cong \mathbb{Z},
\end{gathered}
$$

and the rest of the homology groups are trivial.

\section{The homology of the Milnor fibre}

Once we have computed the homology of $\mathcal{M}$ we can use Proposition 3.1 to compute the homology of the Milnor fibre of $f$.

Since the tubular neighbourhood $T$ is homotopy equivalent to the Milnor fibre of $\Sigma_{s}$ of the 3-dimensional i.c.i.s. $\Sigma_{0}$ we have

$$
\begin{gathered}
H_{0}(T ; \mathbb{Z}) \cong \mathbb{Z}, \\
H_{3}(T ; \mathbb{Z}) \cong \mathbb{Z}^{\mu_{0}}, \\
H_{i}(T ; \mathbb{Z})=0
\end{gathered}
$$

for any other $i$. 
Running the Mayer-Vietoris exact sequences used in order to compute the higher homology of $\mathcal{M}$, we obtain easily that the inclusion of $\mathcal{M}$ in $T$ gives clearly an isomorphism in the $H_{i}$ when $n \geq 7$ and $i \leq 3$. Hence we have the vanishing $H_{i}(T, \mathcal{M} ; \mathbb{Z})=0$ for $1 \leq i \leq 4$, and the isomorphism $H_{i+1}(T, \mathcal{M} ; \mathbb{Z}) \cong H_{i}(\mathcal{M} ; \mathbb{Z})$ for $i \geq 4$.

We have obtained:

Theorem 10.1. Let $\mu_{0}$ and $\mu_{1}$ be the Milnor numbers of the i.c.i.s. $\left(g_{1}, \ldots, g_{n-3}\right)$ and $\left(\operatorname{det}(H(f)), g_{1}, \ldots, g_{n-3}\right)$. The homology of the Milnor fibre is the following:

- If $\operatorname{corank}(H(f)(0) \geq 3$ :

$$
\begin{gathered}
H_{n-1}\left(\mathbf{F}_{f} ; \mathbb{Z}\right) \cong \mathbb{Z}^{\mu_{0}+2 \mu_{1}-4 a+1+\# A_{1}}, \\
H_{k}\left(\mathbf{F}_{f} ; \mathbb{Z}\right)=0
\end{gathered}
$$

if $1 \leq k \leq n-2$,

$$
H_{0}\left(\mathbf{F}_{f} ; \mathbb{Z}\right) \cong \mathbb{Z}
$$

- If $\operatorname{corank}(H(f)(0)=2$ :

$$
\begin{gathered}
H_{n-1}\left(\mathbf{F}_{f} ; \mathbb{Z}\right) \cong \mathbb{Z}^{\mu_{0}+2 \mu_{1}-4 a+2+\# A_{1}} \\
H_{n-2}\left(\mathbf{F}_{f} ; \mathbb{Z}\right) \cong \mathbb{Z} \\
H_{k}\left(\mathbf{F}_{f} ; \mathbb{Z}\right)=0
\end{gathered}
$$

if $1 \leq k \leq n-3$,

$$
H_{0}\left(\mathbf{F}_{f} ; \mathbb{Z}\right) \cong \mathbb{Z}
$$

- If $\operatorname{corank}(H(f)(0)=1$ :

$$
\begin{gathered}
H_{n-1}\left(\mathbf{F}_{f} ; \mathbb{Z}\right) \cong \mathbb{Z}^{\mu_{0}+2 \mu_{1}}, \\
H_{n-3}\left(\mathbf{F}_{f} ; \mathbb{Z}\right) \cong \mathbb{Z}, \\
H_{k}\left(\mathbf{F}_{f} ; \mathbb{Z}\right)=0
\end{gathered}
$$

if $k=n-2$ and if $1 \leq k \leq n-4$,

$$
H_{0}\left(\mathbf{F}_{f} ; \mathbb{Z}\right) \cong \mathbb{Z}
$$

- If $\operatorname{corank}(H(f)(0)=0$ :

$$
\begin{gathered}
H_{n-1}\left(\mathbf{F}_{f} ; \mathbb{Z}\right) \cong \mathbb{Z}^{\mu_{0}}, \\
H_{n-4}\left(\mathbf{F}_{f} ; \mathbb{Z}\right) \cong \mathbb{Z}, \\
H_{k}\left(\mathbf{F}_{f} ; \mathbb{Z}\right)=0 \\
\text { if } k=n-2, n-3 \text { and } 1 \leq k \leq n-4, \\
H_{0}\left(\mathbf{F}_{f} ; \mathbb{Z}\right) \cong \mathbb{Z} .
\end{gathered}
$$


Proof. Our computations work for the case corank $(H(f)(0))>0$, if $n \geq 8$. In order to remove this restrictions we notice that by Thom-Sebastiani theorem the Milnor fibre of $f+z^{2}$ with $z$ a new variable is the suspension of the original Milnor fibre, and that the case corank $(H(f)(0))=0$ was proved by Némethi in [15].

\section{The homotopy type of the Milnor fibre}

Once we have computed the homology of the Milnor fibre, we will use it to recover its homotopy type.

Proposition 11.1. The Milnor fibre $\mathbf{F}_{f}$ is simply connected if $\operatorname{corank}\left(h_{i, j}(0)\right) \neq 0$.

Proof. Since the critical set is of dimension 3, the Kato-Matsumoto bound [10] tells us that the Milnor fibre is $(n-5)$-connected. Thus, if $n \geq 6$ we have that the Milnor fibre $\mathbf{F}_{f}$ is simply connected. For the case where $n=5$, we will need the following reasoning.

Let $Z_{1}, \ldots, Z_{\# A_{1}}$ be representatives of the vanishing cycles of $\mathbf{F}_{f}$ corresponding to the $A_{1}$ points that appear outside $\Sigma_{s}$ in a generic deformation. Let $C\left(Z_{i}\right)$ denote the cone over $\mathcal{Z}_{i}$. Let $C(\pi)$ be the cylinder of the mapping

$$
\pi: \mathcal{M} \rightarrow \Sigma_{s} .
$$

The space $C(\pi)$ is simply connected because it admits the simply connected space $\Sigma_{s}$ as a deformation retract.

By construction we have that

$$
\mathbf{F}_{f} \cup C(\pi) \bigcup_{i=1}^{\# A_{1}} C\left(\mathcal{Z}_{i}\right)
$$

is homotopy equivalent to the contractible space $X_{S}$ (see Section 3). Since each $Z_{i}$ is homeomorphic to $\mathbb{S}^{4}$, by Seifert-Van Kampen theorem, the gluing of the $C\left(Z_{i}\right)$ has no effect over the fundamental group, since both $\pi_{1}\left(C\left(\mathbb{S}^{4}\right)\right)$ and $\pi_{1}\left(\mathbb{S}^{4}\right)$ are trivial. The same reasoning tells us that, if $\pi_{1}(\mathcal{M})$ is trivial, so must be $\pi_{1}\left(\mathbf{F}_{f}\right)$.

The space $\mathcal{M}$ is obtained from $\mathcal{X}$ by gluing the preimage by $\pi$ of several Lefschetz thimbles. These pieces are topologically $D^{3} \times \mathbb{S}^{1}$ glued along $\mathbb{S}^{2} \times \mathbb{S}^{1}$. By SeifertVan Kampen theorem, if $\pi_{1}(\mathcal{X})$ is trivial, the aditios of these pieces does not change the fundamental group. So, to prove that $\pi_{1}(\mathcal{M})=0$ it is enough to prove that $\pi_{1}(\mathcal{X})=0$.

We may compute $\pi_{1}(\mathcal{X})$ using Seifert-Van Kampen with the decomposition

$$
X=\mathscr{B} \cup \mathcal{A}_{1} \cup \cdots \cup \mathcal{A}_{a} .
$$


In Section 5 it is shown that each of the pieces of the above decomposition is, up to homotopy, a fibration over the corresponding piece of the following decomposition:

$$
\Sigma_{s} \cap \operatorname{det}\left(H\left(F_{s}\right)\right)^{-1}(0)=B_{0} \cup A_{1}(\zeta) \cup \cdots \cup A_{a}(\zeta) .
$$

The fibres are always spheres of dimension at least 2. Using this it is easy to see that the computation of $\pi_{1}(\mathcal{X})$ by Seifert-Van Kampen mimics the computation of $\pi_{1}\left(\Sigma_{s} \cap \operatorname{det}\left(H\left(F_{S}\right)\right)^{-1}(0)\right)$, but this space is simply connected (in fact a bouquet of 2-spheres).

We now have all the necessary ingredients to prove our Bouquet Theorem.

Theorem 11.2. The Milnor fibre of a singularity over a 3-dimensional i.c.i.s. with finite extended codimension has the homotopy type of a bouquet of spheres of number and dimensions as in Theorem 10.1.

Proof. From Proposition 11.1 we know that the Milnor fibre is simply connected.

In the case where corank $H(f)(O) \geq 2$ (that is, $a \neq 0$ ) we have computed the integer homology, getting that $\left.H_{n-1}\left(\mathbf{F}_{f} ; \mathbb{Z}\right)\right)$ and $H_{n-2}\left(\mathbf{F}_{f} ; \mathbb{Z}\right)$ are free and finitely generated and $H_{i}\left(\mathbf{F}_{f} ; \mathbb{Z}\right) \cong 0$ otherwise. In this situation, since the Milnor fibre has the homotopy type of a $(n-1)$-complex, we can apply [15], 2.2, and [15], 2.3, and we get the result.

If corank $(H(f)(O)=0$ the result is covered by Theorem 4.1 of [15].

We are left with the case in which $\operatorname{corank}(H(f)(O)=1$. By Criterion 2.2 in [15], we only need to represent each generator of the non-zero homology groups by a chain modelled in a sphere. When corank $(H(f)(O)=1$, in the decomposition of $\mathcal{M}$ given in Section 5 we have that $\mathscr{B}$ coincides with $\mathcal{X}$, that $B_{u}$ is diffeomorphic to $B_{0}$, which are Milnor fibres of the 2-dimensional i.c.i.s. $\Sigma_{0} \cap V(\operatorname{det}(H(f)))$ and that the fibration (11) becomes a homotopy $\mathbb{S}^{n-3}$-fibration

$$
\varphi: \mathcal{X} \rightarrow B_{0} \cong \mathscr{B}_{u} .
$$

The generator of $H_{n-3}\left(\mathbf{F}_{f} ; \mathbb{Z}\right)$ is the Gysin lift of the generator of $H_{0}(B ; \mathbb{Z})$, and hence it is represented by a sphere. By homology splitting, the generators of $H_{n-1}\left(\mathbf{F}_{f} ; \mathbb{Z}\right)$ come from two different places:

- those coming from the $A_{1}$-singularities of $f_{s}$ outside $\Sigma_{s}$, which are clearly represented by spheres (the vanishing cycles of the $A_{1}$-singularities).

- Those coming from $H_{n-1}(\mathcal{M} ; \mathbb{Z})$, which come in turn from two different places:

(1) the ones coming from the image of $H_{n-1}(X ; \mathbb{Z})$ in $H_{n-1}(\mathcal{M} ; \mathbb{Z})$, which are Gysin-liftings over the vanishing cycles of $B_{u}$ of the fibration (32).

(2) Those coming from the addition to $\mathcal{X}$ of the spaces $\mathcal{C}_{i}$ (see the decomposition formula (23)). Recall that each $\ell_{i}$ is the product of a Lefschetz thimble associated to a vanishing cycle of $B_{u}=\left\{\operatorname{det}\left(H\left(f_{s}\right)=u\right\} \cap \Sigma_{s}\right.$ with the homotopy-sphere $\mathbb{S}^{n-4}$, which is the fibre of the fibration (21). 
In the rest of the proof we will represent the cycles of types (1) and (2) by spheres. In order to start we claim that the fibration of $(n-3)$-spheres over $B_{0}$ is trivial.

Let us prove the claim. Since $B_{0}$ is a bouquet of 2 -spheres given by vanishing cycles it is enough to prove that the fibration, restricted to each of the vanishing cycles of $B_{0}$ is trivial. Choose a vanishing cycle $C_{i}$. Move the parameter $s$ so that $s$ is very close to a parameter $s_{0}$ in which $\Sigma_{s} \cap V\left(\operatorname{det}\left(\left(f_{s}\right)\right)\right)$ acquires an $A_{1}$ singularity to which the vanishing cycle $C_{i}$ collapses. In this situation a local change of coordinates shows that to prove that the fibration is trivial over $C_{i}$ is equivalent to prove that the fibration of $(n-3)$-spheres associated to the function

$$
f=\left(x_{1}^{2}+x_{2}^{2}+x_{3}^{2}\right) x_{4}^{2}+\sum_{i=5}^{n} x_{i}^{2}
$$

is trivial over the vanishing cycle of the restriction of $x_{1}^{2}+x_{2}^{2}+x_{3}^{2}$ to $V\left(x_{4}, \ldots, x_{n}\right)$. Proving this is an easy local computation.

Let us represent the generators of type (1). By the claim the group $H_{n-1}(\mathcal{X} ; \mathbb{Z})$ is generated by chains of the form

$$
\tau: \mathbb{S}^{2} \times \mathbb{S}^{n-3} \rightarrow \mathcal{X} \subset \mathcal{M} \subset \mathbf{F}_{f}
$$

where $\tau\left(\mathbb{S}^{2} \times \mathbb{S}^{n-3}\right)$ is a Gysin lift of a vanishing cycle $C_{i}$ of $B_{0}$ by the fibration (32).

Choose a section $s$ of this fibration such that $s\left(C_{i}\right)$ is inside $\tau\left(\mathbb{S}^{2} \times \mathbb{S}^{n-3}\right)$. For $n=5$, the sphere $s\left(\mathbb{S}^{2}\right)$ is trivial in $H_{2}(X ; \mathbb{Z})$, since this group is generated by the fibre. This implies that it is also zero in $H_{2}\left(\mathbf{F}_{f} ; \mathbb{Z}\right)$, and, by Hurewicz's Theorem, it is also trivial in $\pi_{2}\left(\mathbf{F}_{f}\right)$. For $n>5$ the triviality of $s\left(\mathbb{S}^{2}\right)$ in $\pi_{2}\left(F_{t}\right)$ holds by the connectivity of the Milnor fibre. This means that $s\left(\mathbb{S}^{2} \times\{\right.$ point $\left.\}\right)$ can be killed by a 3-disc inside $\mathbf{F}_{f}$. By Lemma 4.5 of [15], we have that the homology class $\left[\tau\left(\mathbb{S}^{2} \times \mathbb{S}^{n-3}\right)\right]$ can be represented by a sphere of dimension $(n-1)$.

We deal now with cycles of type (2). The space $C_{i}$ is the product of a Lefschetz thimble $L_{i}$ associated to a vanishing cycle $C_{i}$ of $B_{u}$ with the sphere $\mathbb{S}^{n-4}$, which is the homotopy-fibre of the fibration (21). Recall that over $B_{u}$ we have in fact a fibration of pairs with fibre homotopic to $\left(\mathbb{S}^{n-3}, \mathbb{S}^{n-4}\right)$ being $\mathbb{S}^{n-4}$ embedded as the equator of $\mathbb{S}^{n-3}$. Consider a collar $K \cong \partial L_{i} \times[0,1]$ of $\partial L_{i}$ in the 3-cell $L_{i}$. We deform continuously the chain given by the embedding of $L \times \mathbb{S}^{n-4}$ in $\mathcal{M}$ so that fibrewise $\mathbb{S}^{n-4}$ is the equator of $\mathbb{S}^{n-3}$ over any point of the internal boundary of the collar and so that $\mathbb{S}^{n-4}$ is collapsed to the north pole of $\mathbb{S}^{n-3}$ at the external boundary $\partial L_{i}$ of the collar. The resulting chain is called

$$
\varphi: L_{i} \times \mathbb{S}^{n-4} \rightarrow \mathbf{F}_{f}
$$

The mapping

$$
s: \partial L_{i} \rightarrow \mathcal{X} \subset \mathbf{F}_{f}
$$


which assigns to a point of $\partial L_{i}$ the north pole of the fibre $\mathbb{S}^{n-3}$ has been seen before to be the trivial element in $\pi_{2}\left(\mathbf{F}_{f}\right)$. Therefore there exists a 3-disk $L^{\prime}$ bounding $\partial L_{i}$ and an extension

$$
s^{\prime}: L^{\prime} \rightarrow F_{t}
$$

of $s$. The identification $L \bigcup_{\partial L_{i}} L^{\prime}$ along their common boundary is a 3-sphere. A representative of our homology class is given by the chain

$$
\psi:\left(L_{i} \bigcup_{\partial L_{i}} L^{\prime}\right) \times \mathbb{S}^{n-4} \rightarrow F_{t}
$$

defined by $\left.\psi\right|_{L \times \mathbb{S}^{n-4}}:=\varphi$ and $\left.\psi\right|_{L^{\prime} \times \mathbb{S}^{n-4}}:=s^{\prime} \circ \mathrm{pr}_{1}$, where $\mathrm{pr}_{1}$ is the projection of $L^{\prime} \times \mathbb{S}^{n-4}$ to the first factor. Notice that the source of $\psi$ is a product of spheres, which we view as a trivial fibration of $\mathbb{S}^{n-4}$ over $L \bigcup_{\partial L_{i}} L^{\prime} \cong \mathbb{S}^{3}$, and that $\psi$ factorises through the result of collapsing to a point the fibre over any point of $L^{\prime}$. Again Lemma 4.5 in [15] represents the homology class by a sphere.

\section{Examples}

Despite the apparent simplicity of the homotopy type of the Milnor fibre of the class singularities considered in this paper, it is possible to find among them unexpected topological behaviours which at the moment have not been observed in singularities with smaller critical set. As an illustration of this we summarise here the properties of a family of examples, which fall in the general class studied in this paper, and which was used in [5] to produce counterexamples to several old equisingularity questions.

Example 12.1. Let $\varphi$ a possibly identical to 0 convergent power series in a variable $x_{1}$. Define

$$
f_{\varphi}:\left(\mathbb{C}^{5}, O\right) \rightarrow \mathbb{C}
$$

by

$$
f_{\varphi}\left(x_{1}, x_{2}, x_{3}, y_{1}, y_{2}\right):=f=\left(y_{1}, y_{2}\right) \cdot\left(\begin{array}{cc}
x_{3} & x_{2} \\
x_{2} & \varphi\left(x_{1}\right)-x_{3}
\end{array}\right) \cdot\left(\begin{array}{l}
y_{1} \\
y_{2}
\end{array}\right) .
$$

If $\varphi$ is not identical to 0 the function $f_{\varphi}$ is of finite codimension with respect to the ideal $I=\left(y_{1}, y_{2}\right)$. The critical set $\Sigma=V\left(y_{1}, y_{2}\right)$ is 3-dimensional and smooth. It is easily checked that the $I$-unfolding

$$
F_{\varphi}:=f_{\varphi}+\sum_{i=0}^{\operatorname{ord}(\varphi)-2} t_{i} x_{1}^{i} y_{2}^{2}
$$


where $\operatorname{ord}(\varphi)$ denotes the order of the series $\varphi$ in $x_{1}$, is the versal $I$-unfolding of $f_{\varphi}$ in the sense of [16] and [4]. Hence we can obtain all $I$-unfoldings of $f_{\varphi}$ by considering deformations of the form

$$
\varphi+\sum_{i=0}^{\operatorname{ord}(\varphi)-2} t_{i} x_{1}^{i}
$$

Notice that the determinant

$$
\operatorname{det} H\left(f_{\varphi}\right)=x_{3}\left(\varphi\left(x_{1}\right)-x_{3}\right)-x_{2}^{2}:(\Sigma, O) \rightarrow \mathbb{C}
$$

has a singularity at the origin of type $A_{2 \operatorname{ord}(\varphi)-1}$. An easy computation shows that if $\left(f_{\varphi}\right)_{s}$ is a generic deformation of $f_{\varphi}$ in its versal $I$-unfolding, the cardinality of the set $\Sigma[2]_{s}$ of points where $H\left(f_{2}\right)$ has corank precisely 2 is equal to $\operatorname{ord}(\varphi)$.

It is also easy to check that for any $s$ in the base of the versal $I$ unfolding the critical set of $f_{s}$ is equal to $\Sigma=V\left(y_{1}, y_{2}\right)$. Hence there are no $A_{1}$ points popping out of $\Sigma$ in a generic $I$-unfolding of $f_{\varphi}$.

Noticing that corank $\left(H\left(f_{\varphi}\right)\right)(O)=2$ we may apply Theorem 10.1 to show that the Milnor fibre is 2-connected, with third Betti number equal to 1 and fourth Betti number equal to

$$
b_{4}=\mu_{0}+2 \mu_{1}-4 a+2+\# A_{1}=0+2(2 \operatorname{ord}(\varphi)-1)-4 \operatorname{ord}(\varphi)+2+0=0 \text {, }
$$

which, surprisingly, is independent of $\varphi$. By Theorem 11.2 we conclude that the Milnor fibre of $f_{\varphi}$ is homotopy equivalent to a 3 -sphere. The remarkable fact is that the homotopy type of the Milnor fibre is independent on $\varphi$ and at the same time the topology of the pair of germs

$$
\left((\Sigma, O),\left(\Sigma[1]_{s}, O\right)\right)
$$

depends heavily on the value $s$ in the base of the versal unfolding.

In [5] it is shown that in fact the diffeomorphism type of the Milnor fibration of the germ $f_{\varphi}$ and the generic Lê-numbers are independent of $\varphi$. Using that the topology of the pair (34) depends on $s$ it is also proven that the topology of the abstract link of $f_{\varphi}$ does depend on $\varphi$. This kind of examples and their stabilisations are at the moment the only known families of examples with constant Lê numbers and constant Milnor fibration and changing topological type. They answer negatively a question of D. Massey in [12]. In [5] modifications of these examples are also used to give the first known counterexample of Zariski's Question B of [24]. Also in [5] these examples were used to construct a family of reduced projective hypersurfaces with constant homotopy type and changing topological type (therefore most classical algebro-topological invariants cannot detect the change in topology). 
Vol. 88 (2013) Topology of hypersurface singularities with 3-dimensional critical set

\section{References}

[1] V. I. Arnold, S. M. Gusein-Zade and A. N. Varchenko, Singularities of differentiable maps. Vol. II. Monodromy and asymptotics of integrals. Monogr. Math. 83, Birkhäuser, Basel 1988. Zbl 0659.58002 MR 0966191

[2] W. Ebeling, The monodromy groups of isolated singularities of complete intersections. Lecture Notes in Math. 1293, Springer-Verlag, Berlin, 1987. Zbl 0683.32001 MR 0923114

[3] J. Fernández de Bobadilla, Approximations of non-isolated singularities of finite codimension with respect to an isolated complete intersection singularity. Bull. London Math. Soc. 35 (2003), 812-816. Zbl 1043.32015 MR 2000028

[4] J. Fernández de Bobadilla, Relative morsification theory. Topology 43 (2004), 925-982. Zbl 1052.32025 MR 2061213

[5] J. Fernández de Bobadilla, Answers to some equisingularity questions. Invent. Math. 161, (2005), 657-675. Zbl 1082.32019 MR 2061213

[6] J. Fernández de Bobadilla, On homotopy types of complements of analytic sets and Milnor fibres. In Topology of algebraic varieties and singularities, Contemp. Math. 538, Amer. Math. Soc., Providence, RI; Real Sociedad Matemática Española, Madrid 2011, 363-367. Zbl 1214.14005 MR 2777829

[7] T. Gaffney, Invariants of $D(q, p)$ singularities. In Real and complex singularities, Contemp. Math. 459, Amer. Math. Soc., Providence, RI, 2008, 13-22. Zbl 1157.32025 MR 2444390

[8] H. Hamm and Lê Dung Trang, Local generalisations of Lefschetz-Zariski theorems. $J$. Reine Angew. Math. 389 (1988), 157-189. Zbl 0646.14012 MR 0953670

[9] T. de Jong, Some classes of line singularities. Math. Z. 198 (1998), 493-517. Zbl 0628.32028 MR 0950580

[10] M. Kato and Y. Matsumoto, On the connectivity of the Milnor fiber of a holomorphic function at a critical point. In Manifolds-Tokyo 1973 (Proc. Internat. Conf., Tokyo, 1973), University of Tokyo Press, Tokyo 1975, 131-136. Zbl 0309.32008 MR 0372880

[11] E. Looijenga, Isolated singular points on complete intersections. London Math. Soc. Lecture Note Ser. 77, Cambridge University Press, Cambridge 1984. Zbl 0552.14002 MR 0747303

[12] D. Massey, The Lê varieties II. Invent. Math. 104 (1991), 113-148. Zbl 0727.32015 MR 1094048

[13] J. Milnor, Singular points of complex hypersurfaces. Ann. of Math. Stud. 61, Princeton University Press, Princeton, NJ, 1968. Zbl 0184.48405 MR 0239612

[14] A. Némethi, The Milnor fiber and the zeta function of the singularities of type $f=P(h, g)$. Compositio Math. 79 (1991), 63-97. Zbl 0724.32020 MR 1112280

[15] A. Némethi, Hypersurface singularities with 2-dimensional critical locus. J. London Math. Soc. 59 (1999), 922-938. Zbl 0949.32012 MR 1709088

[16] R. Pellikaan, Finite determinacy of functions with non-isolated singularities. Proc. London Math. Soc. (3) 57 (1988), no. 2, 357-382. Zbl 0621.32019 MR 0950595

[17] M. Shubladze, Isolated hypersurface singularities of the transversal type $A_{1}$. Bull. Georgian Acad. Sci. 153, (1996), no. 1, 7-10. Zbl 0881.32016 MR 1651960 
[18] D. Siersma, Isolated line singularities. In Singularities, Part 2 (Arcata, Calif., 1981), Proc. Sympos. Pure Math. 40, Amer. Math. Soc., Providence, RI, 1983, 485-496. Zbl 0514.32007 MR 0713274

[19] D. Siersma, Hypersurfaces with singular locus a plane curve and transversal type $A_{1}$. In Singularities (Warsaw, 1985), Banach Center Publ. 20, PWN, Warsaw, 1988, 397-410. Zbl 0662.32011 MR 1101856

[20] D. Siersma, The vanishing topology of non isolated singularities. In New developments in singularity theory (Cambridge, 2000), NATO Sci. Ser. II Math. Phys. Chem. 21, Kluwer Academic Publishers, Dordrecht 2001, 447-472. Zbl 1011.32021 MR 1849319

[21] D. Siersma, bouquet theorem for the Milnor fibre. J. Algebraic Geom. 4 (1995), 51-66. Zbl 0817.32015 MR 1299004

[22] M. Tibar, Bouquet decomposition of the Milnor fibre. Topology 35 (1996), no. 1, 227-241. Zbl 0848.32031 MR 1367282

[23] A. Zaharia, Topological properties of certain singularities with critical locus a 2-dimensional complete intersection. Topology Appl. 60 (1994), 153-171. Zbl 0812.32017 MR 1302470

[24] O. Zariski, Open questions in the theory of singularities. Bull. Amer. Math. Soc. 77 (1971), 481-489. Zbl 0236.14002 MR 0277533

Received February 19, 2010

Javier Fernández de Bobadilla, Universidad Autónoma de Madrid, Instituto de Ciencias Matemáticas, Campus Cantoblanco, UAM, c/ Nicolás Cabrera, 13-15, Spain

E-mail: javierbobadilla73@gmail.com

Miguel Marco-Buzunáriz, Universidad Autónoma de Madrid, Instituto de Ciencias Matemáticas, Campus Cantoblanco, UAM, c/ Nicolás Cabrera, 13-15, Spain

E-mail:mmarco@unizar.es 